\title{
Prediction of the 3D Structure and dynamics of Human DP G- Protein Coupled Receptor bound to an agonist and an antagonist
}

\author{
Youyong Li, Fangqiang Zhu, Nagarajan Vaidehi ${ }^{*}, 1$, and William A. Goddard III \\ Materials and Process Simulation Center (MC 139-74), California Institute of Technology, Pasadena \\ CA 91125
}

Felix Sheinerman, Stephan Reiling, Isabelle Morize, Lan Mu, Keith Harris, Ali Ardati, and Abdelazize Laoui

Sanofi-Aventis Pharma, Bridgewater, NJ 08807-0800

\begin{abstract}
Prostanoids play important physiological roles in the cardiovascular and immune systems and in pain sensation in peripheral systems through their interactions with eight G-Protein Coupled Receptors. These receptors are important drug targets, but development of subtype specific agonists and antagonists has been hampered by the lack of 3D structures for these receptors. We report here the 3D structure for the human DP G-Protein Coupled Receptor (GPCR) predicted by the MembStruk computational method. To validate this structure we use the HierDock computational method to predict the binding mode for the endogenous agonist (PGD2) to DP. Based on our structure, we predicted the binding of a new family of antagonists which has been confirmed experimentally.

We find that PGD2 binds vertically to DP in the TM1237 region with the $\alpha$ chain toward the extracellular (EC) region and the $\omega$ chain toward the middle of the membrane. This structure explains the selectivity of the DP receptor and the residues involved in the predicted binding site correlate very well with available mutation experiments on DP, IP, TP, FP, and EP subtypes. We report molecular dynamics of DP in explicit lipid and water and find that the binding of the PGD2 agonist leads to correlated rotations of helices of TM3 and TM7, whereas binding of antagonist leads to no such rotations. Thus these motions may be related to the mechanism of activation.
\end{abstract}

\section{Introduction}

Prostanoids (prostaglandins (PG) and thromboxanes (TX), both metabolites of arachidonic acid) ${ }^{1,2}$ play important physiological roles in the cardiovascular and immune systems, and in pain sensation in peripheral systems. They exert a variety of actions in the body through binding to specific cell surface prostanoid receptors. The eight subtypes of prostanoid receptors all belong to the family A of G-Protein coupled receptors (GPCRs). Various prostanoids exert their activity preferentially through prostanoid receptors. For example, the DP receptor preferentially binds prostaglandin PGD2 with substantially more affinity than any of the other prostanoid ligands and PGD2 shows quite a preference to DP receptor with diminished activity on EP3 and no activity on the other prostanoid receptors. ${ }^{1}$ This extensive heterogeneity and preference of the prostanoid receptors is reflected by the remarkable diversity of physiological effects that can be elicited by prostanoids.

Emails: wag@wag.caltech.edu, NVaidehi@coh.org.

${ }^{1}$ Present address: Division of Immunology, Beckman Research Institute of City of Hope, 1500, Duarte Road, Duarte, CA-91010. 
Perhaps the most well known of these effects are those that produce pain, fever and inflammation, which can be relieved through the inhibition of COX-1 and/or COX-2 by aspirin and non-steroidal anti-inflammatory drugs (NSAIDs). ${ }^{3,4}$ Other important effects of the prostanoids involve the vascular, reproductive, bone and immune systems. ${ }^{1}$ Specifically, PGD (2) functions as a mast cell-derived mediator to trigger asthmatic responses. ${ }^{5}$

The development of subtype specific agonists and antagonists has been hampered by the lack of 3D structures for prostanoid receptors. As a first step in providing a structural basis for understanding the activity and selectivity of these receptors, we used the MembStruk computational procedure ${ }^{6-14}$ to predict the structure for the human DP receptor starting from its amino acid sequence. The details for these predictions are in section 2 . Then we used the HierDock computational procedure ${ }^{15-20}$ to predict the binding site for endogenous agonist PGD2 with human DP receptor. The details for these predictions are in section 3 . Then we carry out molecular dynamics in explicit lipid and water to check the binding effect on the protein structure. Finally, we report a new antagonist optimized by our structure.

\section{Methods}

We predicted the three dimensional structure of DP using MembStruk4.1 computational method summarized here.

\subsection{Prediction of the TM regions and hydrophobic centers}

The TM regions were predicted using TM2ndS method described in reference ${ }^{10}$. The input to TM2ndS method was the 43 sequences of prostanoid receptors from various species.

Multiple sequence alignment of the 43 sequences was performed using clustalW. Using the multiple sequence alignment as input, the TM regions were predicted using TM2ndS procedure. ${ }^{10}$ The hydrophobic maximum was chosen as the central residue (referred to as the centroid) for each helix that divides the area under the hydrophobicity curve equally. The centroid for each helix is positioned to be in the same xy plane (the midpoint of the lipid).

\subsection{Prediction of the 3D structure}

Based on the predicted TM regions and the TM centroids, the MembStruk program was used to build and optimize the 3-D structure for the human DP receptor. The steps of MembStruk and the predicted structure are described below.

1. Helix packing-First, canonical $\alpha$-helices were built for each TM domain. These $\alpha$-helix structures were then bundled together as follows. The predicted helix centroid is placed on the xy plane using $x, y$ coordinates based on the low resolution ( $7.5 \AA)$ electron density map of frog rhodopsin. The orientation of each helix about its z axis (the $\chi$ angle) is chosen so that its helical face with the maximum hydrophobic moment points outwards to contact the lipid. In this analysis, we calculate the hydrophobic moment over the full helix but including only the half of the residues that would face outward. Then each helix is tilted about the point at which the central axis intersects the xy plane to match the tilt angles $(\theta, \varphi)$ from frog rhodopsin.

2. Helix bending-Next, molecular dynamics (MD) simulations were performed (200 picoseconds) for each individual helix, allowing the helix to attain its equilibrium structure (in some cases it bends or kinks). Then we chose the structure with the lowest potential energy for each helix and assembled it back into the bundle so that the average axis coincides with the original axis. The side chains were then optimized using SCWRL ${ }^{21,22}$ and the total energy minimized (conjugate gradients). 
3. RotMin-This initial packed structure was minimized and then we allowed the individual packing interactions to optimize as follows. Each helix was independently rotated $(\chi)$ by $+5^{\circ}$ and $-5^{\circ}$, the side chains repositioned using SCWRL, and then all atoms of the bundle optimized. If either new angle was lower, it was selected.

4. Lipid Insertion-At this point we inserted the 7 helix bundle into a lipid framework ending up with 48 lipids molecules arranged as a bilayer. These lipid molecules were optimized using rigid body dynamics.

5. RotScan-Starting from the final RotMin structure, we performed a full 360-degree rotational scan $(\chi)$ on each of the helices in $5^{\circ}$ increments. For each angle, the side chains were re-assigned with SCWRL and full bundle re-minimized. Multiple minima based on energy and interhelical hydrogen bonds were chosen for each helix. Combination of multiple minima for each helix leads to an ensemble of conformations which were then sorted by the number of interhelical hydrogen bonds and then by total energy.

\subsection{Prediction of the Extra-Celluar (EC) and Intra-Cellular (IC) loop structure}

We took the best structure from the previous step and added the three EC and IC loops. We expect the three EC and three IC loops of human DP to be quite flexible and strongly affected by the solvent, which is treated only implicitly in MembStruk. Thus to provide initial loop structures for our MD studies of the DP receptor, we used the alignment of DP with bovine rhodopsin and then homology threaded the DP loops to the crystal structure (1L9H.pdb). Then we carried out minimization and dynamics on the loops with fixed helix bundle atoms.

In the crystal structure of bovine rhodopsin the ECII loop (connecting TM4 and TM5) is closed over the 7-TM barrel, contributing to the binding of 11-cis-retinal. This ECII loop has a disulfide bond to TM3 (C105-C183), which is highly conserved among the rhodopsin super family of GPCRs. Thus, we include this disulfide bond in our loop structures. It is generally believed that the disulfide bond plays critical role in the folding of 7 helices and in the closing of the ECII loop over the 7-TM barrel. ${ }^{23}$ Since the rhodopsin in the crystal study is in the inactive form, it is possible that substantial changes occur in ECII and in other loops upon activation.

\subsection{Molecular Dynamics Simulation}

Since the description of lipid and water in MembStruk is implicit with a skimpy layer of lipid bilayer, we performed molecular dynamics (MD) simulations of the predicted structure of DP receptor with and without ligand for $1 \mathrm{~ns}$ in explicit lipid bilayer and water. We carried out MD simulations using NAMD ${ }^{24}$ including explicit water and a periodically infinite lipid to determine the interactions of the protein with lipid and water. ${ }^{14}$

We started with the predicted hDP structure, stripped away the lipid molecules, and inserted it in a periodic structure of (POPC: 1-palmytoil-2-oleoyl-sn-glycero-3 -Phosphatidylcholine). In this process, we eliminated lipid molecules within $5 \AA$ of the protein. Then we inserted this in a box of water molecules and eliminated waters within $5 \AA$ of the lipid and protein. Then keeping the protein fixed we allowed the lipid and water to relax using minimization. Then we minimized the whole system before doing dynamics. The full system (Figure 4) contains the hDP protein, 100 lipid molecules, 6617 water molecules, 15 chlorine ions for a total of 33,347 atoms per periodic cell. The box size is $66 \AA$ by $66 \AA$ by $72 \AA$. We then used the NAMD program to carry out 2 nanosecond of NPT MD with a bath temperature of $300 \mathrm{~K}$. 


\subsection{HierDock Method: Scan the entire DP receptor for binding sites}

We used HierDock approach to predict the binding mode of ligand to DP receptor. The first step is to scan all void regions (shown as magenta dots in Figure 8) in the entire DP receptor structure to locate putative binding regions for PGD2. The void region in the entire receptor structure was partitioned into 27 regions and the HierDock method was used to dock the cyclopentane ring of PGD2 in each box. Here we examined the best binding sites that have at least $80 \%$ buried surface area. This leads to the TM1237 region shown in Figure 8.

Subsequently we docked the entire PGD2 molecule in this putative binding region using the HierDock2.0 method. The large cavity in the TM1237 region arises from the presence of prolines on TM2 and TM7. The proline on TM7 is 100\% conserved among the rhodopsin super family. The proline on TM2 is highly conserved among prostanoid family except TP and FP, which have a glycine residue before that position. Rhodopsin has two glycines around that position and the bending angle of TM2 helix is in such a shape, which makes TM2 helix is packing tightly with TM1,3,7 helices.

\subsection{Results and discussion}

\subsection{Description of the predicted human DP structure}

The DP receptor sequence lacks some of the well-conserved motifs present in class A GPCRs. For example, the DRY motif on TM3 is ECW, the well-conserved Trp on TM4 becomes Leu, the WXP motif on TM6 becomes SXP and the NPXXY motif on TM7 is a DPWXF in the DP receptor. Thus, we can expect that the DP receptor might have a different set of stabilizing interhelical hydrogen bonds from rhodopsin. The predicted 3D structure of human apo-DP receptor is shown in Figure 2 and the residues forming interhelical H-bonds are highlighted.

We find an interhelical hydrogen bond between N34(1) and D72(2). N34(1) and D72(2) are conserved in the rhodopsin family A including DP, but the conserved Asn of the NPXXY motif in TM7 is a DPWXF motif in the DP receptor. S316(7), which is not a conserved residue, makes a hydrogen bond with the N34(1) and D72(2). D319(7) makes a hydrogen bond with S119(3). D72(2) also forms a strong salt bridge with the K76(2) on the same helix. K76(2) is a conservative replacement in other prostaglandin receptors except for thromboxane receptors. We also find a hydrogen bond between R310(7) and Y87(2), where R310(7) is conserved across all prostaglandin receptors while Y87 is present only in DP receptors.

\subsection{Molecular dynamics study of the predicted human DP structure with lipid and water}

After predicting human apo-DP structure, we performed 1ns of MD simulations on the apo protein structure.

Figure 3 shows the 3D structure of human DP receptor after 1ns MD with lipid and water. We find that the protein remains stable during the simulations. Thus, Figure 5 shows the five important interhelical hydrogen bonds (HB) are all maintained during the 1ns MD.

Of particular interest is that five water molecules diffuse into binding pocket within the TM regions (Figure 6a), forming water mediated hydrogen bonds with the buried polar residues. For example, three water molecules cluster around D319(7), with one water molecule around D72(2) and one around S119(3). Thus the interhelical hydrogen bond between N34(1) and D72 (2) become water mediated. Figure $6 \mathrm{~b}$ shows the time evolution of the distance of these water molecules to the polar residues. The rhodopsin X-ray structure also showed some water molecules sufficiently strongly bound to be observed, ${ }^{24}$ which were observed in the vicinity of highly conserved residues and have been suggested to regulate the activity of rhodopsinlike GPCRs. 
Figure 7a shows the RMSD time evolution of the helical segments during the 1ns MD. Here RMSD is with respect to the last frame of the $1 \mathrm{~ns}$ trajectory. The total RMSDs ranges from the initial predicted structure to the final one in the trajectory ranges for $1.5 \AA$ to $2.3 \AA$. Focusing on the last 200 pico second, these fluctuations range from $1.0 \AA$ to $1.5 \AA$, except TM5 which reaches $2.0 \AA$.

Figure $7 \mathrm{~b}$ shows the time evolution of the loop segments. Larger movements are observed for the loops, with fluctuations ranging from $1.5 \AA$ for ECII (the most rigid loop due to the disulfide linkage with TM3) to $3.5 \AA$ for ICIII. Focusing on the last 200 pico second, we find fluctuations of $1.0 \AA$ for ECII and $2.3 \AA$ for ICIII.

\subsection{Predicted binding site of prostanoid compounds in the predicted DP structure}

The predicted binding site of PGD2 is shown in Figure 9a. PGD2 is located between the TM $1,2,3,7$ helices and is covered by the ECII loop.

We find favorable hydrophobic interactions of the $\alpha$ chain with L26(1) and F27(1). The $\alpha$ chain of PGD2 points up toward the EC region with the $\omega$ chain pointing down between TM1 and TM7. The critical elements of bonding are:

- The carboxylic acid interacts with R310(7).

- The carbonyl on the cyclopentane ring of PGD2 has a hydrogen bond with K76(2).

- The hydroxyl on the $\omega$ chain interacts with S316(7) and K76(2).

- Our predicted structure had no hydrogen bond between S313(7) and 9-OH on the cyclopentane ring of PGD2. However, in the MD simulations we find that a hydrogen bond forms between $\mathrm{S} 313(7)$ and 9-OH.

- A hydrophobic pocket surrounds the $\alpha$ chain with M22(1), G23(1), Y87(2), W182 (ECII), L309(7), R310(7), L312(7), and S313(7) within $6 \AA$.

- A hydrophobic pocket surrounds the $\omega$ chain with L26(1), G30(1), I317(7), P320(7), and W321(7) within $6 \AA$.

\subsection{Molecular dynamics study of the complex of hDP - PGD2 with lipid and water}

After inserting the predicted PGD2/hDP complex into the infinite lipid membrane and solvating fully with water (using the procedure described in section 2.4), we performed $2 \mathrm{~ns}$ of MD. Figure 9 compares the structure of the complex after 2 ns molecular dynamics with the initial predicted structure. We find that the three hydrogen bonds of the original predicted structure remain stable during $2 \mathrm{~ns}$ dynamics. However, one additional H-bond is formed between $9-\mathrm{OH}$ group of PGD2 and S313(7).

Figure 10 shows the time evolution for the hydrogen bond distances between PGD2 and hDP.

- 15-OH--S316(7): the hydrogen bond distance remains mostly between 2.9 and $3.1 \AA$ with occasional extensions to $3.8 \AA$. For the apo-protein, S316 was hydrogen bonded to K76(2)

- 1 -COOH--R310(7): the salt bridge distance is $\sim 3.0 \AA$ for most the time with occasional extensions to $4.0 \AA$.

- $11-\mathrm{C}=\mathrm{O}-\mathrm{K} 76(2)$ : the HB distance is $\sim 3.5 \AA$ for most the time with occasional extensions to $4.0 \AA$ and a brief one to $6.5 \AA$ A.. For the apo-protein, K76 was hydrogen bonded to S316(7) 
- 9-OH--S313(2): the initial distance is $6 \AA$, but it quickly contracts to $3.5 \AA$ for half of the trajectory, then bounces between $4.5 \AA$ and $7 \AA$, and finally comes back to 4 A.

Thus the agonist has disrupted the K76(2)-S316(7) coupling of the apo-protein, leading to the clockwise rotation of TM3 and the countercloskwise rotation of TM7. Thus the changes in interactions between 9-OH, S313(2) and 11-C=O, K76(2) shown in Figure 10 are probably related to activation of the receptor.

As shown in Figure 11, the four important interhelical hydrogen bonds: N34(1)-D72(2), D72 (2)-K76(2), D319(7)-S119(2), Q122(3)-D319(7) are rather rigid, remaining between $2.8 \AA$ to $3.0 \AA$, while the $5^{\text {th }}$ one: K76(2)-S316(7) starts at $\sim 4 \AA$, drops to $3.2 \AA$ for $1 \mathrm{~ns}$, and finally increases rapidly to $\sim 5.5 \AA$. The decoupling between K76(2) and S316(7) occurs because of the binding of $15-\mathrm{OH}$ of PGD2 to S316(7) and 11-C=O to K76(2).

The eta angle evolution in Figure 12(a) shows that over the 2ns of trajectory, TM7 helix (D319 (7) and S316(7)) is rotating anticlockwise and TM3 helix is rotating clockwise in a cogwheel pattern as shown in Figure 12(b). Figuer 12(c) aligned the structure of TM3 and TM7 before $\mathrm{MD}$ and after 2ns MD.

Thus, we conclude that PGD2 forms strong interactions with both TM2 and TM7. The binding of ligand between TM2 and TM7 breaks the strong coupling between TM2 and TM7. This then induces a rotation of TM7. Due to the strong coupling of TM7 with TM3, this in turn induces a rotation in TM3 as shown in Figure 12. This shows how binding of ligand induces changes in other helices that could in turn lead to activation.

This analysis indicates the function of the conserved D319 on TM7. It is not involved directly in the binding of the ligand. But it couples with TM3 to likely play a role in the process of activation. Indeed, based on mutation experiments, Satoh et al. 25 concluded that the conserved Asp on TM7 of EP3 is important for activation, but not for ligand binding.

Our conclusion that D319(7) is important for the activation is consistent with experiments on rhodopsin. ${ }^{26}$ The NPxxY (7) of rhodopsin is analogous to DPWxy where $\mathrm{x}=\mathrm{I} / \mathrm{V}, \mathrm{y}=\mathrm{F} / \mathrm{Y}$ in the prostanoid receptors, where the D is 319 for DP. In rhodopsin, the NPxxY (7) and E/DRY (3) motifs provide, in concert, a dual control of the activation structural changes in the photoreceptor. ${ }^{26}$

In addition, fluorescence experiments on rhodopsin during activation, show that TM3-TM6 interaction are involved for GPCR activation. ${ }^{27,28}$ Our results on DP indicates the role of TM3 in activation, but suggest that it is coupled with TM7 rather than TM6. Thus, TM3-TM7 interaction may pass the signal to intracellular part (ECW motif, analogous to DRY of rhodopsin) to activate the receptor.

In addition, with PGD2 bound to DP, we find that two water molecules (Figuer 13) move into the active site: one to form hydrogen bond with S119(3) and the other to hydrogen bond to D319(7). The other three water molecules observed to form hydrogen bond with D319 and D72 in apo hDP are now blocked by the bound PGD2.

\subsection{The hydrophilic interactions and hydrophobic interactions in the predicted binding mode of PGD2 in DP receptor}

Figure 9 shows that the important hydrogen bonds between the agonist PGD2 and $h$ DP. These are:

- $1-\mathrm{COOH}$ of the $\alpha$ chain with R310(7) and W182(ECII). 
- $\quad 15-\mathrm{OH}$ of the $\omega$ chain with S316(7) and K76(2).

- $11-\mathrm{C}=\mathrm{O}$ of the cyclopentane ring to $\mathrm{K} 76(2)$

- $\quad 9-\mathrm{OH}$ of the cyclopentane ring to $\mathrm{S} 313(7)$.

In addition there are favorable hydrophobic channels with residues interacting with the hydrophobic parts of the $\alpha$ and $\omega$ chains: $\alpha$ chain with M22(1), G23(1), L26(1), F27(1), V83 (2), Y87(2), W182(ECII), L309(7), L312(7), $\omega$ chain with G30(1), L31(1), S313(7), S316(7), $\mathrm{I} 317(7), \mathrm{P} 320(7)$, and W321(7).

\subsection{Contributions to binding of each TM region; comparison with mutation results}

3.6.1 TM7-The residues of TM7 predicted to play a role in hydrogen bonding to PGD2 are: R310, S313, and S316, while hydrophobic interactions include L309, L312, I317, P320, and W321.

Particularly important is the salt bridge of R310 with the carboxylic acid part of the $\alpha$ chain of PGD2. Strong evidence in support of this interaction is that mutation of this Arg on IP, 30,31 $\mathrm{EP} 2,{ }^{32} \mathrm{EP} 3,{ }^{34,35} \mathrm{FP},{ }^{37}$ and $\mathrm{TP}^{38}$ leads to significant loss of binding. Such mutation experiments have not yet been carried out for the $h \mathrm{DP}$ receptor. S313 and S316 are forming hydrogen bonds with PGD2 in our predicted binding mode. Also no experiments have been reported for the effect of mutations in S313 or S316 on binding to PGD2. Supporting evidence for interactions of PGD2 with L309 (interacting with the $\alpha$ chain) and R310 is that mutation of the analogous residues F278 and R279 in the IP receptor lead to a dramatic decrease in binding of iloprost. ${ }^{30,31}$ (wild type: 7.9nM; F278A: 351.3nM; R279A: >500nM)

F311 and V314 face the lipid in our predicted structure and are not involved in binding PGD2. This is consistent with experimental observations for the IP receptor, that mutations of the corresponding residues F280 and F283 30,31 do not affect binding. (F280A 10.8nM; F283A: $6.2 \mathrm{nM}$ ) We find that L312 of DP interacts directly with the $\alpha$ chain of PGD2. This is consistent with experiments by Kedzie et al ${ }^{32}$ who found that the mutation of the analogous residue L304 of EP2 to Y enhances iloprost potency by 100-fold. Although we find R310 to be directly involved in binding, we find that L306 and V314 are close to the bound ligand but do not interact with it directly. This is consistent with experiments by Funk et al. ${ }^{38}$ who studied mutation of the analogous residues R295, L291, and W299 on TM7 of TP receptor, and showed that W299R, R295Q, and L291F mutations lead to loss of binding to agonist I-BOP. (Four experiments with data not shown in the paper). Moreover, W299L binds to agonists I-BOP and U-46619.

We find that D319(7) makes a hydrogen bond with S119(3) on TM3 as discussed in section 2 and is not involved in binding to PGD2. D319(7) is 100\% conserved among prostanoid receptors and is believed to be important for activation. This aspartic acid residue corresponds to Asn(7) in the NPxxY motif of bovine rhodopsin and might couple with TM2 and/or TM3 upon signal transduction. Mutation studies on the residue analogous to D319(7) in EP3 25, 34 and FP 37 support its expected role for signal transduction and the absence of role in ligand binding.

3.6.2 TM2-K76(2) on TM2 is predicted to hydrogen bond to the with $\mathrm{C}=\mathrm{O}$ of the cyclopentane ring of PGD2 and 15-OH hydroxy group on the $\omega$ chain of PGD2. K76(2) in turn is also strongly coupled to D72(2) via a salt bridge. We predict that K76(2) is key in distinguishing PGD2 from other prostaglandins. Evidence in favor of this interaction is that the chimera $\boldsymbol{D} \boldsymbol{P}_{\boldsymbol{N}-\boldsymbol{I}} / \boldsymbol{I} \boldsymbol{P}_{I I-E X I} / \boldsymbol{D P} \boldsymbol{P}_{I I I-C} 29,39$ recognizes iloprost and not PDG2. Moreover, $\mathrm{CR}_{\mathrm{T} 94 \mathrm{~K}}$ (CR: Chimeric Receptor) acquires the ability to bind PGD2, Ki 23nM compared to $11 \mathrm{nM}$ for $\mathrm{mDP}$ while retaining its binding to iloprost. 
Favorable hydrophobic interactions are provided by S80(2), V83(2), and Y87(2). This is consistent with experiments on the FP receptor, where H81(2)/FP corresponds to K76(2)/DP. Rewald et al. ${ }^{36}$ found that substitution of H81(2) of rat FP with a number of different amino acids led either to a loss of ligand binding or to alterations in the optimum $\mathrm{pH}$ for receptor ligand interaction. Moreover Neuschafer-Rube et al. ${ }^{37}$ found that $\mathrm{H} 81 \mathrm{~A}(2)$ of FP abolishes ligand binding. (from 6.4nM to no binding) Our predicted structure has Q89(2) facing lipid and forming hydrogen bond with the backbone of A85(2) and L96(ECI). This is consistent with the mutation result that the chimeric receptor $\mathrm{CR}_{\mathrm{R} 107 \mathrm{Q}}{ }^{29,39}$ does not change the binding of PGD2.

Residues L78, V82, L84, A85, and R91 on TM2 of DP lie farther than 6 A from the bound PGD2, and do not participate directly in binding. L78, V82, L84, and A85 face the lipid, while R91 faces TM1, but is not close to bound PGD2. Y87 covers PGD2 and R91 is one turn above Y87. This is consistent with the observation that CRT94K binds to both PGD2 and iloprost

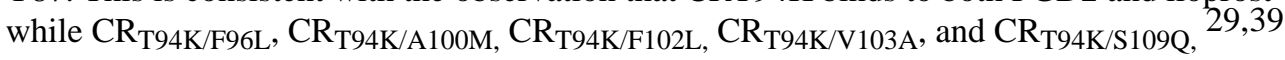
do not change the binding to PGD2. We observe that S80 and Y87 of DP have favorable hydrophobic interactions with the $\alpha$ chain of PGD2 and evidence in favor of this comes from studies on IP which is the closest prostanoid receptor to DP. Mutations by Stitham et al. ${ }^{30}$, 31 on human prostacyclin receptor of the analogous residues $\mathrm{S} 68 \mathrm{~A}(2)$ and $\mathrm{Y} 75 \mathrm{~A}(2)$ lead to significant change of binding affinity of iloprost (wild type: 7.9nM; S68A: 62.3nM; Y75A: $>500$ ). We predict that D72(2) couples with 15-OH of PGD2 through K76(2) while also interacting with N34(1). This is consistent with the observation that D72(2) is 100\% conserved among all the rhodopsin superfamily while the $15-\mathrm{OH}$ of the $\omega$ chain is $100 \%$ conserved among all prostanoid compounds. In addition mutation by Stitham et al. ${ }^{30,31}$ on human prostacyclin receptor of the corresponding residue D60(2) leads to significant change of binding affinity of iloprost.(from $7.9 \mathrm{nM}$ to $>500 \mathrm{nM}$ )

3.6.3 TM1-In the predicted structure of the $h$ DP receptor, TM1 provides hydrophobic stabilization to the $\alpha$ chain of PGD2 with M22, G23, L26, F27, G30, L31, and N34. There is limited mutation data on TM1 of prostanoid family receptors. Evidence that G23(1) is directly involved in the binding pocket was provided by Kobayashi et al. ${ }^{29,39}$ who studied chimeras of the mouse prostaglandin I receptor (mIP) and the mouse prostaglandin D receptor (mDP). mIP recognizes iloprost and PGE1, while mDP recognizes only PGD2. However, the chimera $\boldsymbol{D} \boldsymbol{P}_{\boldsymbol{N}-\boldsymbol{I}} / \boldsymbol{I P}_{\boldsymbol{I I - E X I}} / \boldsymbol{D P} \boldsymbol{P}_{I I I-C}$ recognizes only iloprost. $\mathrm{CR}_{\mathrm{G} 22 \mathrm{~S}}$ of the chimera recovers the ability to bind PGE1, PGE2 and PGD2. We find that A19 faces the lipid, close to TM2, and not involved in binding PGD2. Evidence supporting this is that $\mathrm{CR}_{\mathrm{A} 19 \mathrm{P}} 29,39$ does not change the binding of PDG2. L26 and L31 are directly involved in the predicted binding mode and the chimera $\mathrm{CR}_{\mathrm{L} 25 \mathrm{M} / \mathrm{L} 30 \mathrm{~V}} 29,39$ does not bind to PGD2 and iloprost.

3.6.4 TM3 -F108, M112, and F115 on TM3 provide hydrophobic stabilization to the $\alpha$ chain and the cyclopentane ring of PGD2. Evidence in favor of this is that Stitham et al. ${ }^{30,31}$ found that F95A(3) and F97A(3) of IP (corresponding to F108(3) and F110(3) of DP) change the binding affinity of iloprost greater than 10 fold. F108(3) interacts with both the $\alpha$ chain and $\omega$ chain of PGD2 while F110(3) of DP is located between TM2 and TM4, which might play a role in stabilizing the helix bundle.

3.6.5 ECII-We predict that PGD2 is located within the TM1237 region and covered by the ECII loop. W182 in ECII forms a hydrogen bond to the 1-COOH of PGD2 and T181 is within $6 \AA$ of PGD2. Both T181 and W182 on ECII are conserved among prostanoid family receptors. EP2/EP4 chimera studies by Stillman et al. ${ }^{40,41}$ show that residues on ECII play a role in ligand binding and that the conserved threonine of ECII is required for iloprost ligand binding. Other studies by Audoly et al. ${ }^{33}$ who examined binding of EP3 to prostanoid compounds with a C-1 methyl ester, show that ECII is involved in prostanoid binding. They found that mutations 
on ECII EP3 $3_{\mathrm{W} 199 \mathrm{~A}}, \mathrm{EP}^{\mathrm{T} 202 \mathrm{~A}}$ (analogous to $\mathrm{C} 178$ and T181 in DP) resulted in increased in

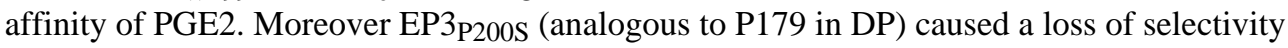
$<20$ fold.

These residues are within $6 \AA$ of the ligand in the predicted binding mode.

Ruan et al. combined NMR, mutation, and modeling to study loop structure and binding modes of TP and IP. ${ }^{42,43}$ They conclude that the ligand could be presented into two binding pockets, ligand recognition pocket (loop region) and TM pocket. This might explains the selectivity of the receptor.

\subsection{Functional Groups in the prostanoid compounds that confer selectivity to prostanoid receptors}

The prediction that the carboxylic acid group of PGD2 interacts with R310(7) is confirmed strongly by various experiments. The carboxylic acid group and the hydroxyl group on the $\omega-$ chain are present in all the prostanoid compounds. R310(7) is 100\% conserved among prostanoid receptor family and K76(2) is not. Other hydrophobic residues P320(7), W321(7) interacting with $\omega$ chain are also $100 \%$ conserved in prostanoid receptor family. Structure activity relationship studies of PGE2 show that the carboxylic acid group, both the $\omega$ chain itself and the hydroxyl group in the $\omega$ chain are critical for agonist activity. ${ }^{44}$ The hydroxyl and carbonyl groups on the cyclopentane ring are not present in all prostanoid compounds and these groups offer receptor selectivity to the ligand as discussed next.

DP receptor binds to PGD2 and PGD1 with similar affinity, and shows at least 2 orders of magnitude lower affinity to other prostanoid compounds. However the IP receptor binds to PGE1 and PGI analogs (iloprost), but it does not bind PGE2. Assuming that these other prostanoid compounds bind to the $h \mathrm{DP}$ receptor in similar binding mode as PGD2, we can explain the how the DP receptor prefers PGD2 to other prostanoid compounds like PGF2 $\alpha$, PGE2, and iloprost.

Figure 15 shows the PGD2, PGF2 $\alpha$, PGE2, and Iloprost prostanoid compounds along with the numbering convention. The difference among prostanoid compounds is in the functional groups and their location on the cyclopentane ring. The predicted binding site for PGD2/DP has a hydrogen bond of the 11- $\mathrm{C}=\mathrm{O}$ carbonyl group on the cyclopentane ring to K76(2). K76 (2) is only present in DP, and the 11-C=O carbonyl is present only in PGD2. PGF2 $\alpha$, PGE2, and iloprost share the 15-OH hydroxyl group, but they have 11-OH hydroxyl group instead of 11-CO on the cyclopentane ring. More importantly, the 11-position hydroxyl group on PGF2 $\alpha$, PGE2, and iloprost points opposite to the 15-OH hydroxyl group both lying on opposite sides of the plane of the cyclopentane ring. As a result the 11-OH hydroxyl group does not have a favorable interaction for PGF2 $\alpha$, PGE2, and iloprost with the DP receptor. K76(2) of the DP receptor has a much stronger interaction with PGD2 than PGF2 $\alpha$, PGE2, and iloprost. Figure 16 shows this unfavorable interaction between K76(2) and 11-OH on PGE2. Figure 17 compares the hydrogen bond distance between hDP and PGE2 over 1ns of MD. This shows that the conserved 1- $\mathrm{COOH}$ and $15-\mathrm{OH}$ have stable hydrogen bonds, while the $9-\mathrm{C}=\mathrm{O}$ and 11$\mathrm{OH}$ of PGE2 do not form stable hydrogen bonds with hDP receptor.

\subsection{Important residues for binding}

As discussed above, the predicted structure of PGD2/DP is consistent with available experimental results. However many of these experiments on other prostanoid receptors have not been reported for DP. In addition our predicted structure suggests a number of new mutation candidates that can be tested out for determining the binding site location of PGD2 based on the predicted model. These include M22(1), G23(1), L26(1), F27(1), G30(1), L31(1), N34(1), 
D72(2), K76(2), S80(2), V83(2), Y87(2), F108(3), M112(3), F115(3), W182(EC2), L309(7), R310(7), L312(7), S313(7), S316(7), I317(7), P320(7), and W321(7).

\subsection{Developing a new antagonist for the human DP receptor}

As an application of our predicted structure for DP receptor, we illustrate here the use of predicted structures to improve drug design. This led to compounds closely related to one currently undergoing clinical trials.

Sanofi-Aventis identified a new class of pyrimidine antagonists to DP, shown in Figure 18 (and denoted as 19a). This 2-Methoxypyrimidine compound has IC50 $=0.8 \mu \mathrm{M}$ for human DP receptor. We started with 19a and report here some of the predictions for the optimum variation. Independently, Sanofi-Aventis synthesized and measured the IC50 for each one. The best predicted compound 19i was found to have an IC50 1000 time better than the starting compound.

We predicted the best binding structure of 19a with human DP receptor, which identified interactions shown in Figure 18 as most important. We predict this compound to be located in TM1237 region, occupying a binding site similar to the agonist PGD2 (Figure 9).

Ligand 19a has an extended conformation similar to PGD2. We find that it has hydrogen bonds with R310(7), and D72(2), similar to PGD2. However, 19a also has a hydrogen bond to T69 (2). These three residues interact with the head, middle, and tail part of ligand 19a, respectively. On the other hand, 19a does not have the strong interactions with K76(2), S316(7), and S313 (7) exhibited by PGD2.

This predicted binding site provides an explanation of the important interaction of ligand 19a with the receptor. We find four critical regions: pyrimidine ring, Benzene ring $\mathrm{A}$, Benzene ring $\mathrm{B}$, the segment $\mathrm{HN}-\mathrm{CH}_{2}-\mathrm{C}\left(\mathrm{CH}_{3}\right)_{2}$ connecting the pyrimidine ring with benzene ring $\mathrm{B}$.

In our predicted bound structure:

- The methoxy on benzene ring A interacts with R310(7).

- Benzene ring A and pyrimidine ring are located in a hydrophobic cavity that includes L26(1), K76(2), F115(3), S316(7), L312(7), and I315(7).

- The conserved signature residue D72(2) forms a hydrogen-bond (HB) with the proton of the $\mathrm{HN}-\mathrm{CH} 2-\mathrm{C}(\mathrm{CH} 3) 2$ part of the ligand.

- Benzene ring B is located among TM127, interacting with N34(1), L68(2), T62(2), and D319(7).

- O-Methyl on benzene ring B forms an HB with T62(2).

Comparing with the binding mode of PGD2, we see that this antagonist shares a similar binding position. The most significant similarity is that both PGD2 and ligand 19a bind strongly with D72(2) and R310(7). However, 19a does not interrupt the hydrogen bond network among TM127. As discussed in section 3.3, the agonist PGD2 makes hydrogen bonds with both D72 (2) and S316(7) causing a disruption of the coupling between TM2 and TM7, which we suggested is involved in activation. The fact that the antagonist 19a does not disrupt TM127 is consistent our hypothesis about activation by the agonist.

Based on our predicted binding mode, we predicted the binding energies of many modified compounds. Eight of these cases are shown in Figure 19. 
Compound $19 \mathrm{~b}$ has modifications on ring $\mathrm{B}$ and the linker $\mathrm{HN}-\mathrm{CH} 2-\mathrm{C}(\mathrm{CH} 3) 2$. And it has better interactions with S316(7) and D319(7). We predicted that the binding energy is improved by $1.4 \mathrm{kcal} / \mathrm{mol}$. Independently the binding (IC50) of the compound was found to improve a factor of 8 to $104 \mathrm{nM}$.

Compound 19c and 19d have different modifications on ring B. 19c achieves better binding energy and IC50, while 19d binds worse due to van der Waals repulsive interaction.

Compound 19e $\sim 19 \mathrm{i}$ have modifications on ring A. We predict that substitutions with carbonic acid or other similar groups lead to substantial improvements in binding due to favorable interaction with R310(7). These compounds were also found to show increased binding affinity (IC50) as shown in Figure 19. Indeed the best compound 18i from the theory has the best observed binding, with IC $50=0.8 \mathrm{nM}, 1000$ times better than the starting compound 19a. Also the compounds predicted to be $2^{\text {nd }}$ best, $3^{\text {rd }}$ best etc are in exactly the same sequence as in the experiments. A compound closely related to $19 \mathrm{i}$ is currently undergoing clinic trials.

\section{Summary}

The 3D structure for the human DP G-Protein Coupled Receptor (GPCR) predicted using the MembStruk computational method leads to features compatible with general understanding of the prostanoid receptors. For example it has a hydrogen bond coupled triad [Asn34(1), Asp72 (2), Ser316(7)] that is conserved among the rhodopsin superfamily of GPCRs and believed to play a role in activation. We validated the stability of the predicted apo-DP structure with 1 nanosecond of molecular dynamics using an infinite lipid bilayer and explicit water $(\sim 33,000$ atoms/cell). We found that five water molecules diffuse into the active site region but the predicted structure and the predicted inter-helical interactions are stable.

To further validate this structure we used the HierDock computational method to predict the binding site and 3D structure for PGD2 (the endogenous agonist) to DP. The predicted binding site positions the PGD2 in a vertical orientation with the $\alpha$ chain toward the extracellular (EC) region and the $\omega$ chain toward the middle of the membrane. It has the following interactions:

- $1-\mathrm{COO}^{-}$of PGD2 interacting with R310(7),

- $\quad$ 15-OH forming hydrogen bond with both S316(7) and K76(2).

- $11-\mathrm{C}=\mathrm{O}$ interacting with $\mathrm{K} 76(2)$, and

- 9-OH forming an hydrogen bond with S313(7),

In addition, hydrophobic cavities from TM1237 surround the hydrophobic parts of the $\alpha$ and $\omega$ chains. Thus, PGD2 binds in the TM1237 region and is covered by the ECII loop. We validated the stability of the predicted PGD2/DP structure with 2 nanosecond of MD studies using an infinite lipid bilayer and explicit water (33,000 atoms/cell). We found that two water molecules diffuse into the active site region. Most important the ligand-protein interactions 9OH-S313(7), 11C=O- K76(2), and 15OH-S316(7) disrupt the coupling of K76(2) and S316 (7) in the apoprotein, inducing a clockwise rotation of TM3 and counterclockwise rotations in TM7. In contrast binding of the antagonist does not disrupt the of K76(2) - S316(7) coupling so that no such motion occurs. Thus these rotations of TM7 and TM3 might be related to activation.

The predicted binding site of PGD2 in hDP explains the variations in experimental activity among various prostanoid compounds to $\mathrm{hDP}$ and to mutated versions. In addition, the residues involved in the predicted binding site correlate very well with available mutation experiments on IP, TP, FP, and EP subtypes, providing an understanding of the selectivity of prostanoid receptors. Thus R310(7), which is 100\% conserved among the prostanoid family, recognizes 
the 1-carboxylic acid on the $\alpha$ chain (also 100\% conserved for all prostanoids) and S316(7) (one turn above the conserved proline), which is $100 \%$ conserved among the prostanoid family, recognizes the 15 -hydroxyl on the $\omega$ chain (also $100 \%$ conserved for all prostanoids). In addition the K76(2) and S313(7) interact with the cyclopentane ring of PGD2 conferring the selectivity of DP receptor. Particularly, K76, which is present only in DP, recognizes the 11position carbonyl, which is present only in PGD2.

The agreement of the predicted PGD2/DP structure with available experimental data validates the DP and PGD2/DP structures, providing the first atomistic understanding of prostanoid receptors.

To illustrate the value of such structures, we used our predicted structure for the DP receptor to optimize substitutions for a new antagonist. Experimental SAR measurements showed excellent agreement with the predictions, suggesting the MembStruk protein structures are sufficiently accurate for drug development..

\section{Supplementary Material}

Refer to Web version on PubMed Central for supplementary material.

\section{Acknowledgements}

Support for the Caltech activities was provided by Sanofi-Aventis Corporation with additional support from NIH (R21-MH073910-01-A1). The computational resources for these studies were provided by grants from ARO-DURIP and ONR-DURIP.

\section{References}

1. Narumiya S, Sugimoto Y, Ushikubi F. Physiological Reviews 1999;79:1193. [PubMed: 10508233]

2. Breyer RM, Bagdassarian CK, Myers SA, Breyer MD. Annu Rev Pharmacol Toxicol 2001;41:661. [PubMed: 11264472]

3. Marnett LJ, DuBois RN. Annu Rev Pharmacol Toxicol 2002;42:55. [PubMed: 11807164]

4. Subbaramaiah K, Dannenberg AJ. Trends Pharmacol Sci 2003:24. [PubMed: 12498727]

5. Matsuoka T, Hirata M, Tanaka H, Takahashi Y, Murata T, Kabashima K, Sugimoto Y, Kobayashi T, Ushikubi F, Aze Y, Eguchi N, Urade Y, Yoshida N, Kimura K, Mizoguchi A, Honda Y, Nagai H, Narumiya S. science 2000;287:2013. [PubMed: 10720327]

6. Hall SE, Floriano WB, Vaidehi N, Goddard WA III. Chem Senses 2004;29:595. [PubMed: 15337685]

7. Floriano WB, Vaidehi N, Goddard WA III. Chemical Senses 2004;29:269. [PubMed: 15150141]

8. Kalani MY, Vaidehi N, Hall SE, Trabanino R, Freddolino P, Kalani MA, Floriano WB, Kam V, Goddard WA III. Proc Natl Acad Sci 2004;101:3815. [PubMed: 14999101]

9. Freddolino P, Kalani MY, Vaidehi N, Floriano W, Hall SE, Trabanino R, Kam VWT, Goddard WA. Proc Natl Acad Sci 2004;101:2736. [PubMed: 14981238]

10. Trabanino R, Hall SE, Vaidehi N, Floriano W, Goddard WA. Biophys J 2004;86:1904. [PubMed: 15041637]

11. P, H.; N, V.; WB, F.; SE, H.; WA, G. Protein Science 2005;14:703. [PubMed: 15722446]

12. Peng J, Vaidehi N, Hall S, Goddard WA III. ChemMedChem 2006;2006in press

13. Vaidehi N, Schlyer S, Trabanino R, Kochanny M, Abrol R, Koovakat S, Dunning L, Liang M, Sharma S, Fox JM, Floriano WB, Mendonça FLd, Pease JE III, WA G, Horuk R. J Biol Chem 2006;281:27613. [PubMed: 16837468]

14. Spijker P, Vaidehi N, Freddolino P, Hilbers P, Goddard W. Proc Natl Acad Sci 2006;103:4882. [PubMed: 16551744]

15. Datta D, Vaidehi N, Floriano WB, Kim KS, Prasadarao NV, Goddard WA III. Proteins: Structure, Function and Genetics 2003;50:213. 
16. Floriano WB, Vaidehi N, Zamanakos G, Goddard WA III. J Med Chem 2004;47:56. [PubMed: 14695820]

17. Datta D, Vaidehi N, Zhang D, Goddard WA III. Protein Science 2004;13:2693. [PubMed: 15388861]

18. Cho A, Wendel JA, Vaidehi N, Kekenes-Huskey PM, Floriano WB, Maiti PKG, Goddard WA III. J Comp Chem 2005;26:48. [PubMed: 15529328]

19. McClendon C, Vaidehi N, Kam V, Zhang D, Goddard W. Protein Eng Design \& Selection 2006;19:195.

20. Gama C, Tully S, Sotogaku N, Clark P, Rawat M, Vaidehi N, Goddard W, Nishi AH-WLC. Nature Chemical Biology 2006;2:467.

21. Canutescu A, Shelenkov A, Dunbrack R. Protein Science 2003;12:2001. [PubMed: 12930999]

22. Altschul S, et al. Journal of Molecular Biology 1990;215:403. [PubMed: 2231712]

23. Palczewski K, Kumasaka T, Hori T, Behnke C, Motoshima H, Fox B, Trong I, Teller D, Okada T, Stenkamp R, Yamamoto M, Miyano M. science 2000;289:739. [PubMed: 10926528]

24. Okada T, Fujiyoshi Y, Silow M, Navarro J, Landau EM, Shichida Y. Proc Natl Acad Sci 2002;99:5982. [PubMed: 11972040]

25. Satoh S, Chang CS, Katoh H, Hasegawa H, Nakamura K, Aoki J, Fujita H, Ichikawa A, Negishi M. Biochemical and Biophysical Research Communications 1999;255:164. [PubMed: 10082673]

26. Fritze, et al. PNAS 2003;100:2290. [PubMed: 12601165]

27. Farrens D, Altenbach C, Yang K, Hubbell W, Khorana H. science 1996;274:768. [PubMed: 8864113]

28. Sheikh S, Zvyaga T, Lichtarge O, Sakma T, Bourne H. nature 1996;383:347. [PubMed: 8848049]

29. Kobayashi T, Ushikubi F, Narumiya S. J Bio Chem 2000;275:24294. [PubMed: 10827082]

30. Stitham J, Stojanovic A, Merenick BL, O’Hara KA, Hwa J. J Bio Chem 2003;278:4250. [PubMed: 12446735]

31. Stitham J, Stojanovic A, Ross LA, Blount AC, Hwa J. Biochem 2004;43:8974. [PubMed: 15248755]

32. Kedzie KM, Donello JE, Krauss HA, Regan JW, Gil DW. Mol Pharmacol 1998;54:584. [PubMed: 9730918]

33. Audoly L, Breyer R. J Biol Chem 1997;272:13475. [PubMed: 9153190]

34. Audoly L, Breyer RM. Mol Pharmacol 1997;51:61. [PubMed: 9016347]

35. Huang C, Tai HH. Biochem J 1995;307:493. [PubMed: 7733888]

36. Rehwald M, Neuschafer-Rube F, de Vries C, Puschel GP. FEBS Letters 1999;443:357. [PubMed: 10025963]

37. Neuschafer-Rube F, Engemaier E, Koch S, Roer U, Puschel GP. Biochem J 2003;371:443. [PubMed: 12519077]

38. Funk CD, Furci L, Moran N, Fitzgerald GA. Mol Pharmacol 1993;44:934. [PubMed: 8246916]

39. Kobayashi T, Kiriyama M, Hirata T, Hirata M, Ushikubi F, Nakamura K. J Bio Chem 1997;272:15154. [PubMed: 9182536]

40. Stillman BA, Audoly L, Breyer RM. Eur J Pharmacol 1998;357:73. [PubMed: 9788776]

41. Stillman BA, Breyer MD, Breyer RM. Mol Pharmacol 1999:56.

42. Ruan K, Wu J, So S, Jenkins L, Ruan C. Eur J Biochem 2004;271:3006. [PubMed: 15233797]

43. So S, Wu J, Huang G, Huang A, Li D, Ruan C. J Bio Chem 2003;278:10922. [PubMed: 12551898]

44. Ungrin MK, Carriere MC, Denis D, Lamontagne S, Sawyer N, Stocco R, Tremblay N, Metters KM, Abramovitz M. Molecular Pharmacology 2001;59:1446. [PubMed: 11353805]

45. Chiang N, Kan WM, Tai HH. Arch Biochem Biophys 1996;334:9. [PubMed: 8837733]

46. D’Angelo DD, Eubank JJ, Davis MG, Dorn GW II. J Bio Chem 1996;271:6233. [PubMed: 8626415]

47. Dorn GWI. J Bio Chem 1990;265:4240. [PubMed: 2137820] 
Nte: (1) MKSPFYRCQNTTSVE (15)

TM1 : (16) KGNSAVMGGVLFSTGLLGNLLALGLLARS (44)

IC1 : (45) GLGWCSRRPLRPLP (58)

TM2 : (59) SVFYMLVCGLTVTDLLGKCLLSPVVLAAYAQNRS (92)

EC1 : (93) LRVLA (97)

TM3 : (98) PALDNSLCQAFAFFMSFFGLSSTLQLLAMALECWLSLG (135)

IC2 : (136) HPFFYRRHITLRL (148)

TM4 : (149) GALVAPVVSAF SLAFCALPFMGFGKF (174)

EC2: (175) VQYCPGTWCFIQMVHEEGSLS (195)

TM5 : (196) VLGYSVLYSSLMALLVLATVLCNLGAMRNL (225)

IC3: (226) YAMHRRLQRHPRSCTRDCAEPRADGREASPQPLEE (260)

TM6: (261) LDHLLLLALMTVLFTMCSLPVIYRAYYGAFKDVK (294)

EC3: (295) EKNRTSEEAED (305)

TM7: (306) LRALRFLSVISIVDPWIFIIFRSPV (330)

Cte: (331) FRIFFHKIFIRPLRYRSRCSNSTNME SSL (359)

Figure 1.

The TM regions and EC, IC loops of human DP receptor predicted using TMPred. 


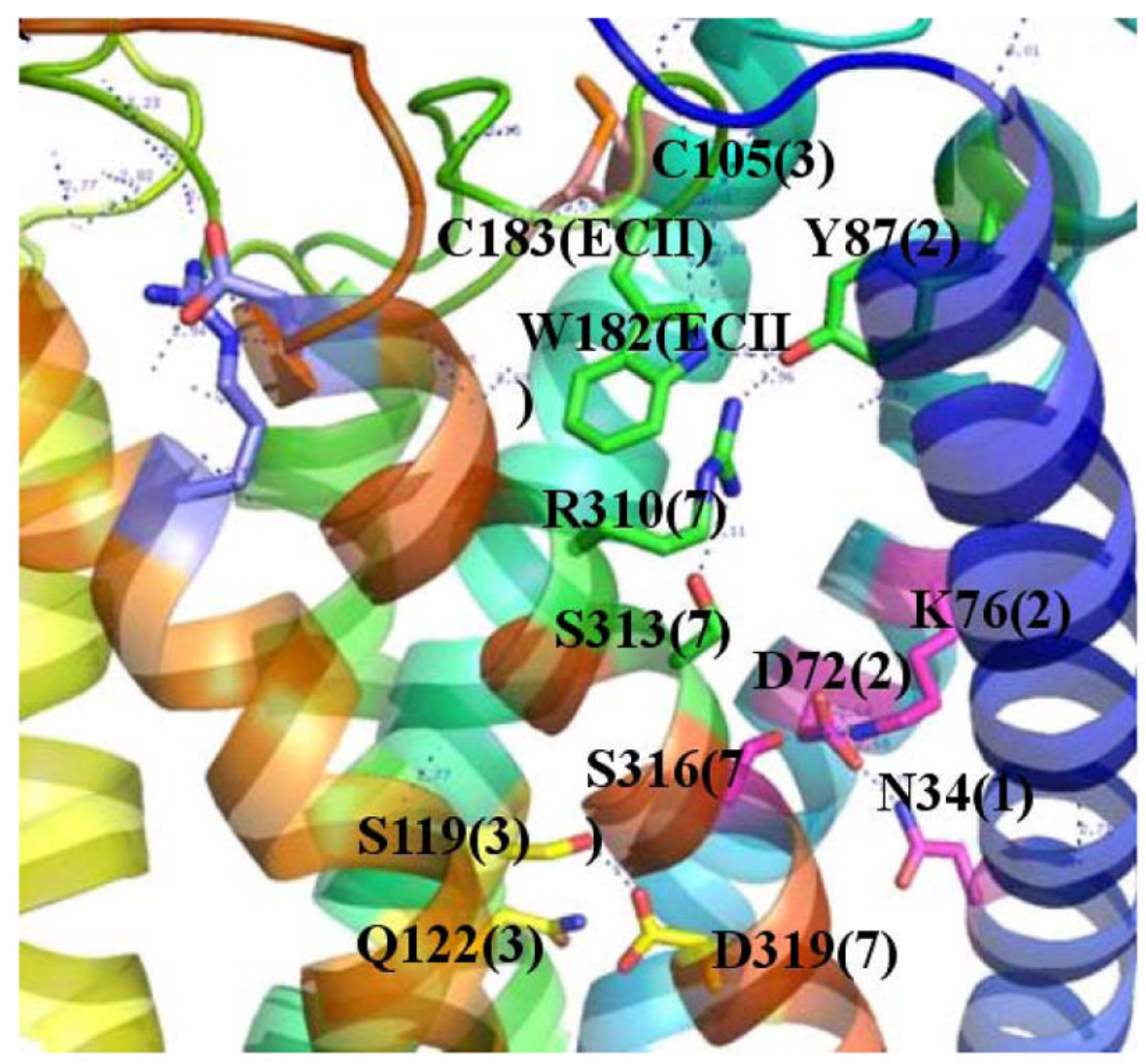

Figure 2.

Predicted 3D structure of human DP receptor from MembStruk. (Residues forming interhelical H-bonds are highlighted here) 


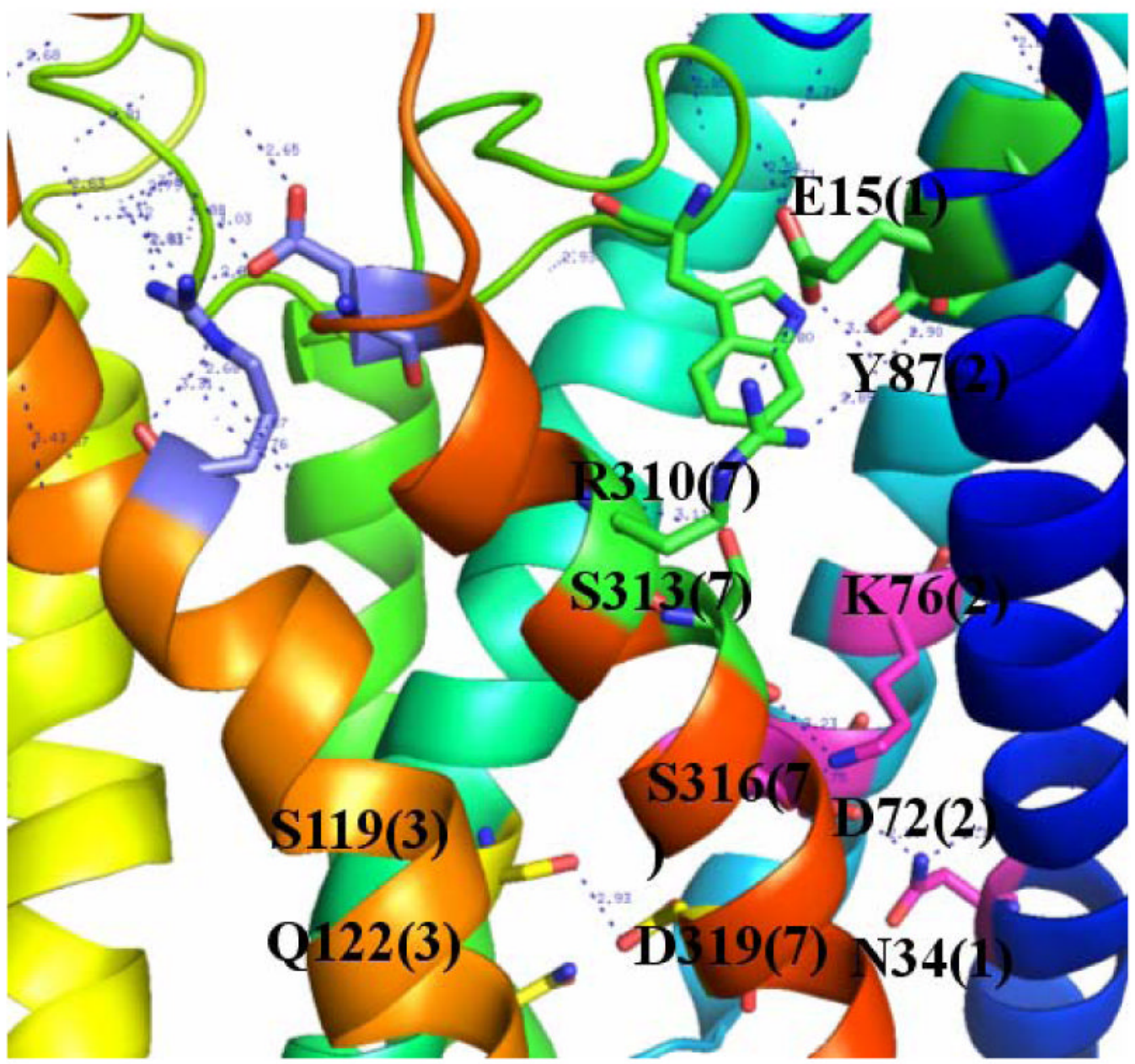

Figure 3.

3D structure of human apo-DP receptor after 1ns MD with lipid and water (Residues forming interhelical H-bonds and water are highlighted and they are stable during 1ns MD) 


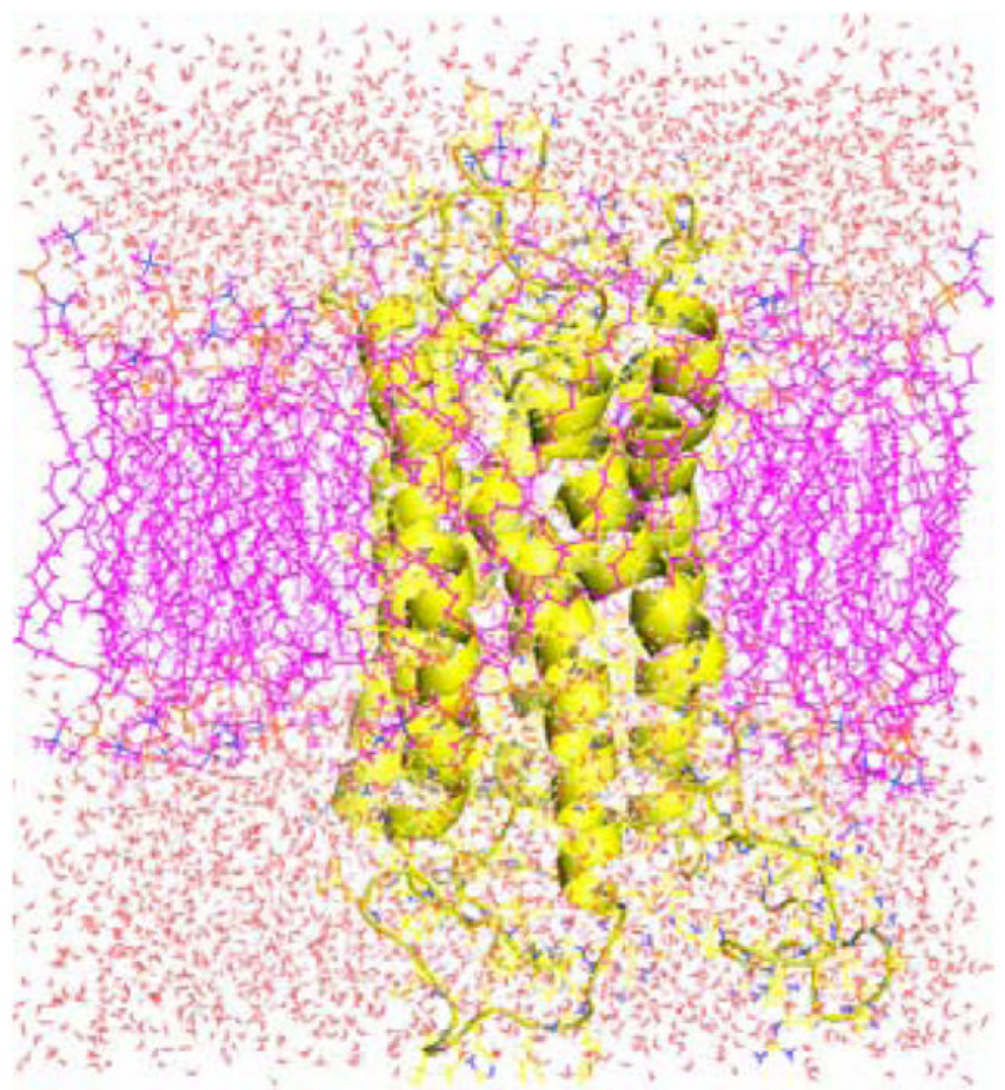

Figure 4.

The molecular dynamics simulation box of hDP with lipid and water. Structure after 1 nanosecond of simulation. The EC region is at the top. 


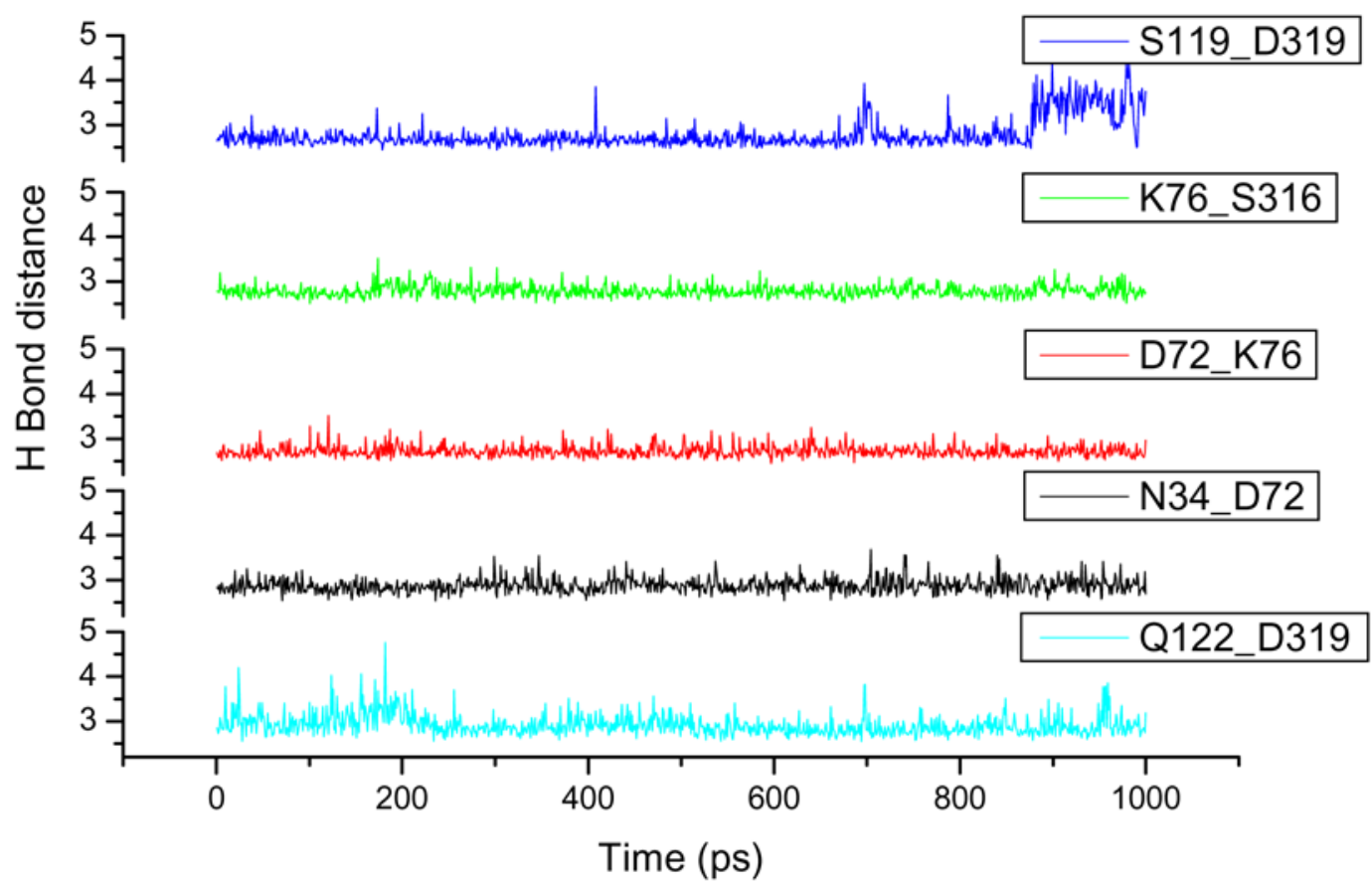

Figure 5.

The stability of the predicted Hydrogen Bonds (HB) in hDP over $1 \mathrm{~ns}$ of MD with full lipid and water. This shows that the Hydrogen Bonds are conserved during MD 


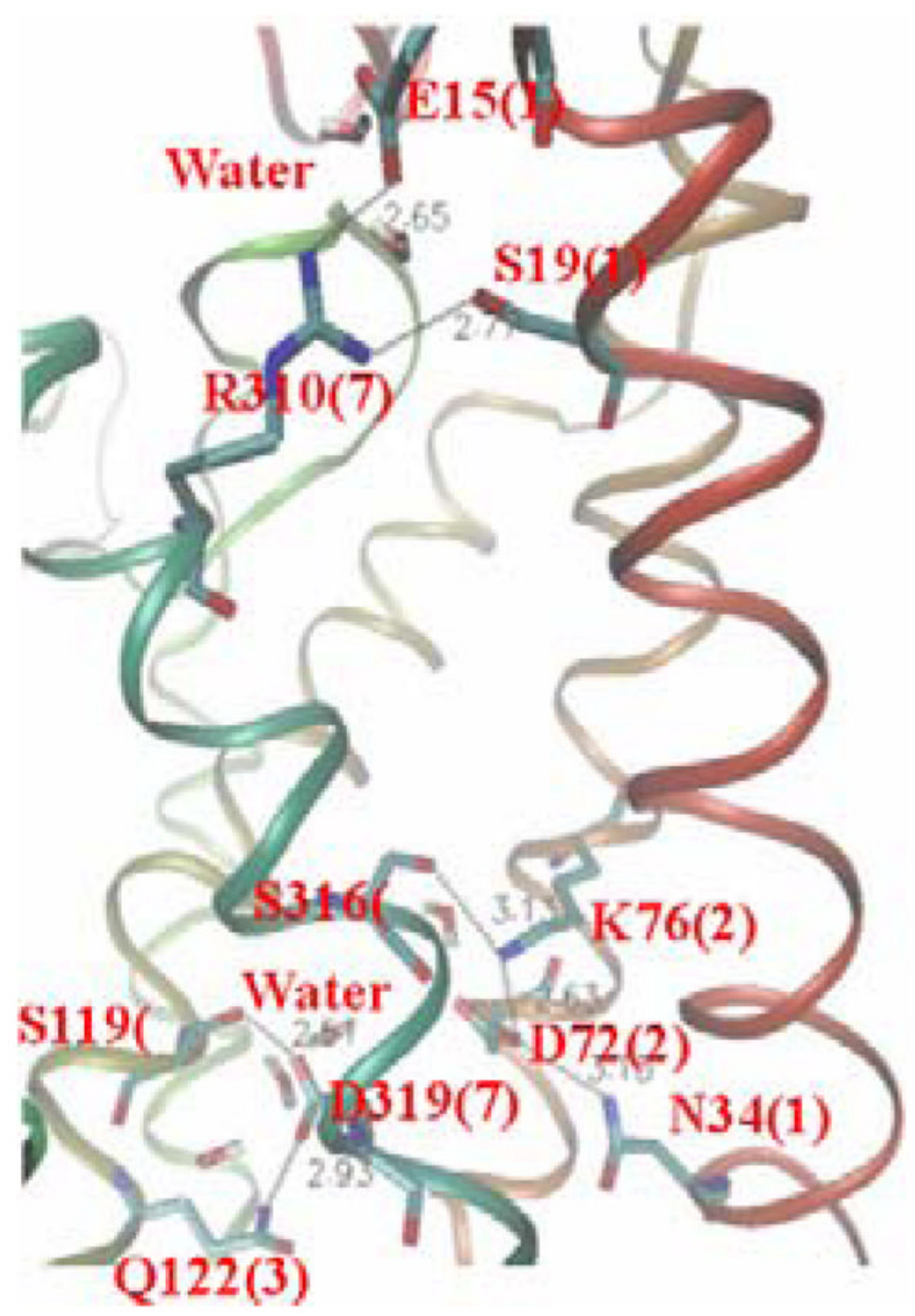

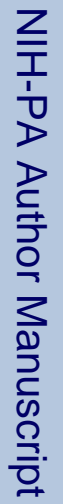




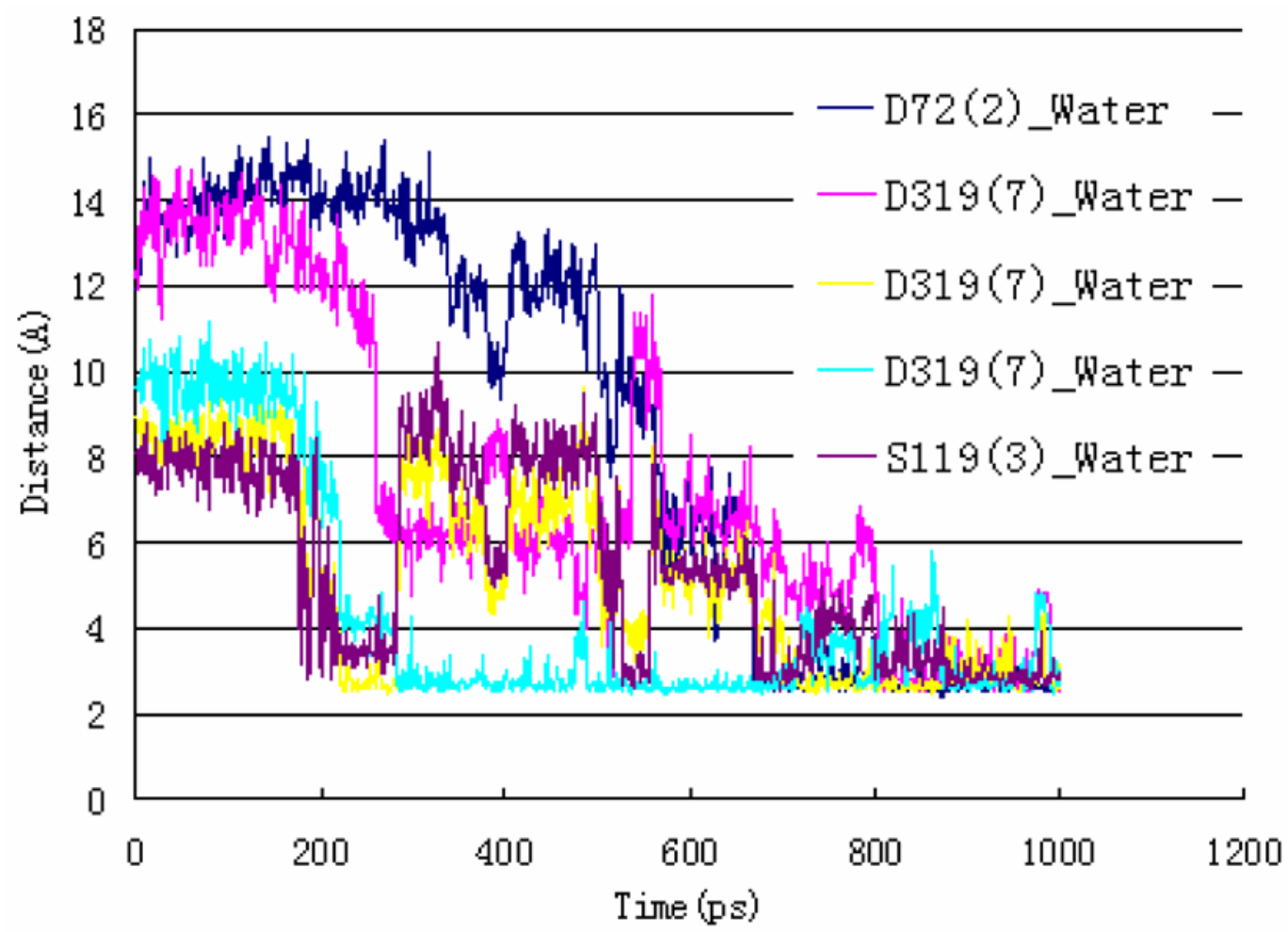

Figure 6.

Figure 6a. 3D structure of human apo-DP receptor after 1ns MD with lipid and water (water and residues forming interhelical Hydrogen bonds are highlighted).

Figure $6 \mathrm{~b}$. The final structure for hDP finds 5 waters in the binding site. This shows the evolution of the distance of the waters to the hydrophilic residues in the pocket. 

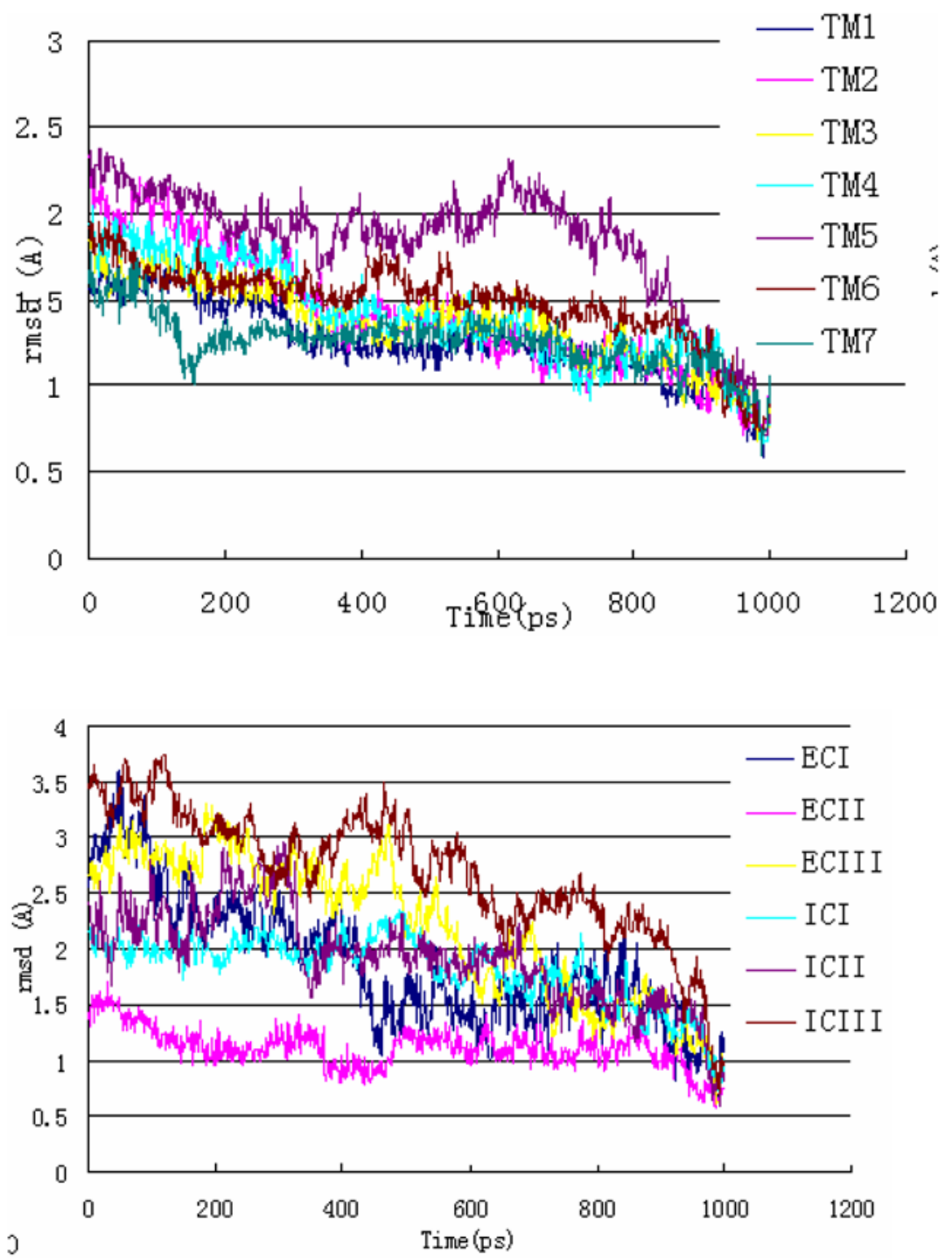

Figure 7.

RMSD evolution of each helix and loop during 1ns molecular dynamics (the reference is the last frame). ECII is rigid due to the disulfide linkage with TM3. 


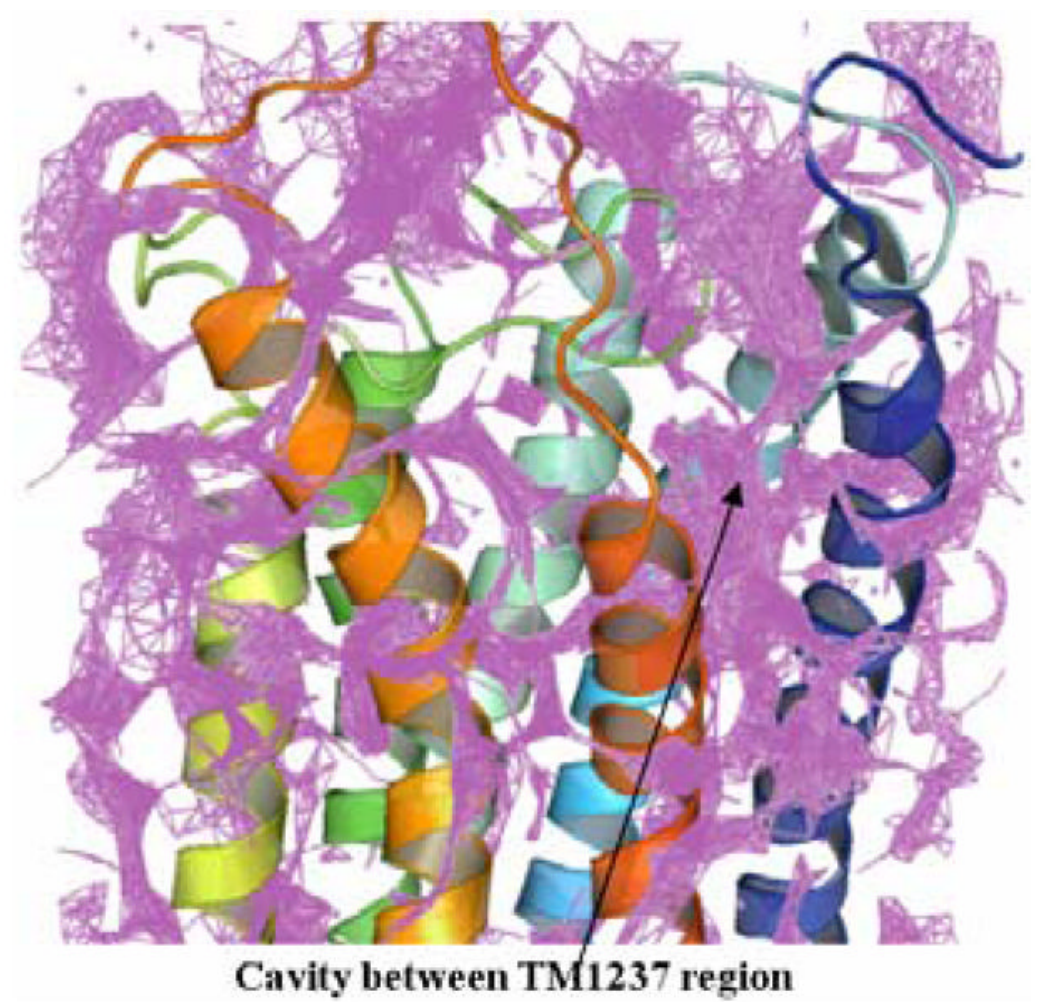

Figure 8.

Void regions of the human DP receptor from a scan using the cyclopentane ring of PGD2. Spheres are shown in magenta dots. The best binding site is obtained in the TM1237 region. 


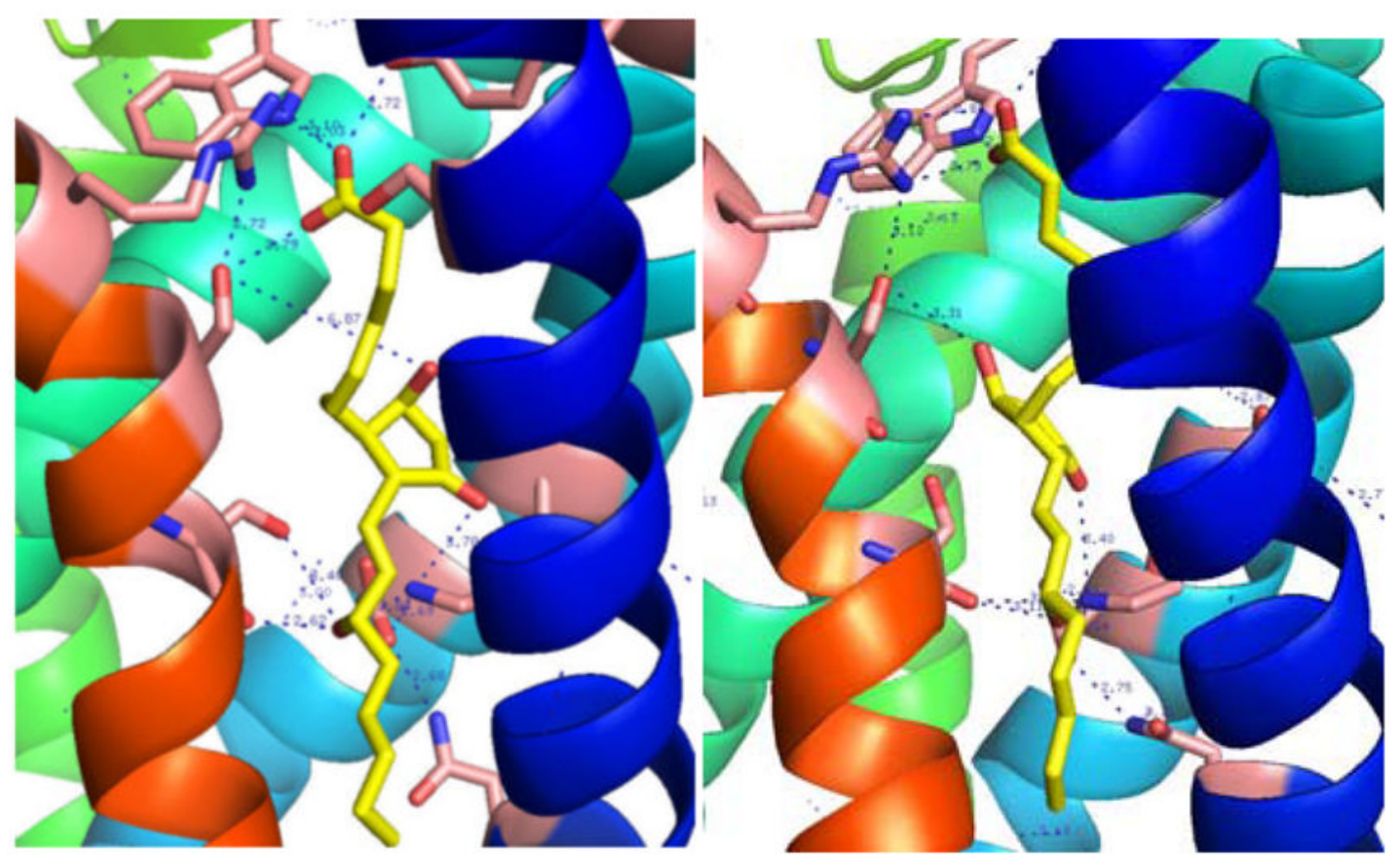

Figure 9.

a) Initial predicted binding site of PGD2 in the DP structure. b) The final binding site after 2ns MD with lipid and water. The essential elements of the binding mode are retained but additional favorable interactions are found. 


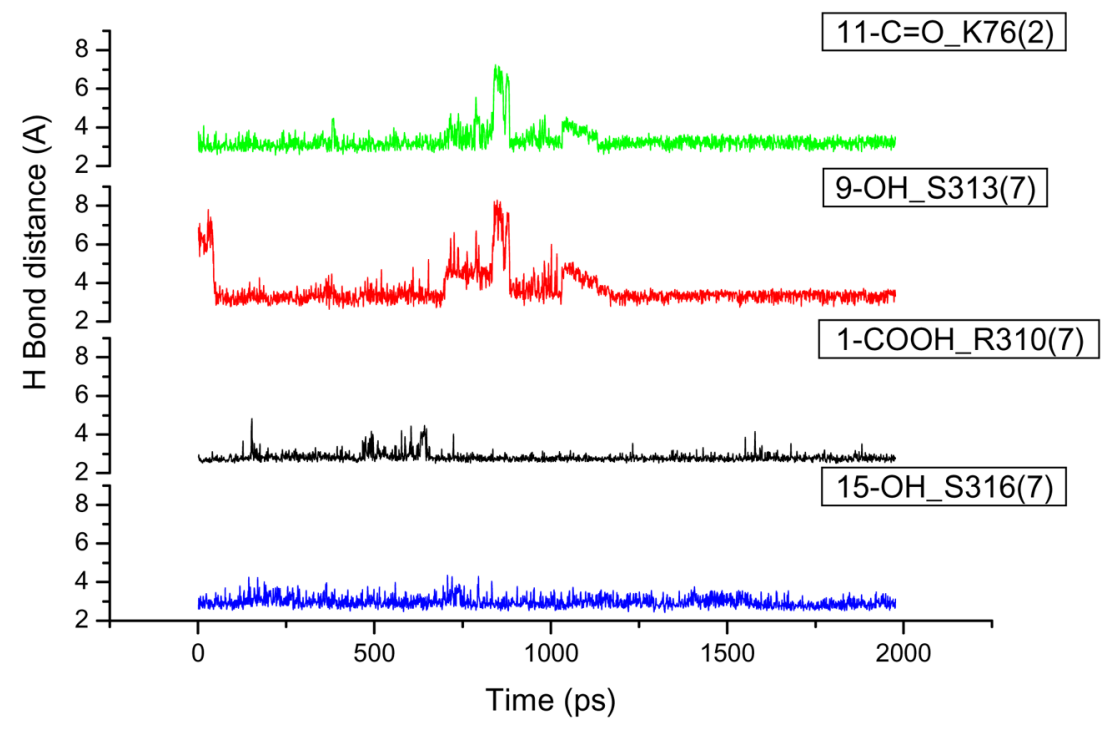

Figure 10.

The H-bond distances bonding PGD2 to hDP remain stable during the 2ns Molecular Dynamics with lipid and water. Note in particular the formation of the 9-OH--S313(7) hydrogen bond not present in the original predicted structure. The 1-COOH--R310(7) and 15-OH--S316(7) anchors remain stable. We see more fluctuations in the interactions of the cyclopentane ring with some breaking and forming of hydrogen bonds to TM2 and TM7. This is related to the fluctuation of hydrogen bond between K76(2) and S316(7) as shown in Figure 11 and the activation of the receptor. 


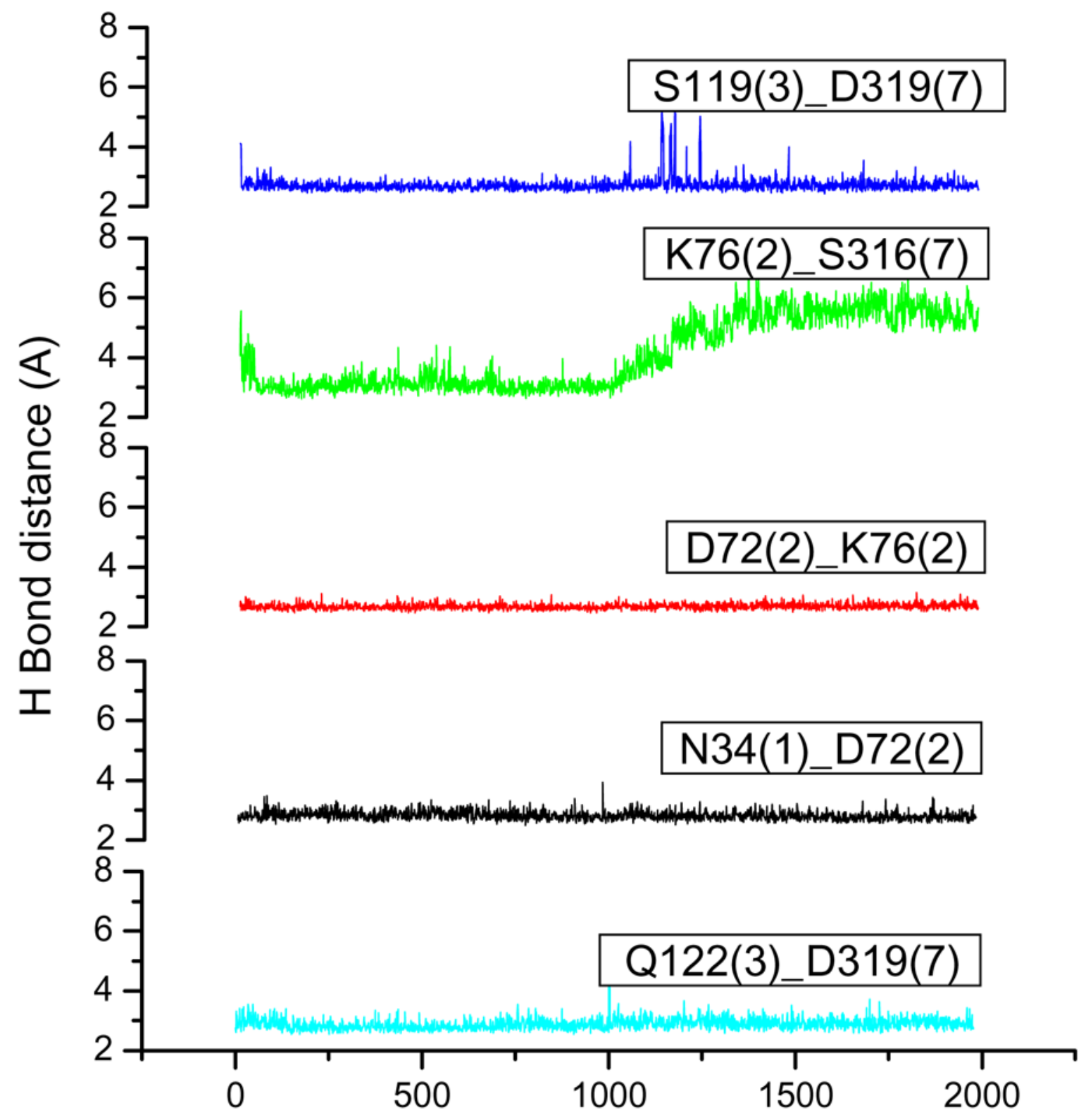

Figure 11.

The inter-helical hydrogen bond distances inside hDP remain stable during 2ns MD with lipid and water, except the hydrogen bond between K76(2) and S316(7). The breakage is the effect of binding ligand, which is presented between TM2 and TM7. The tight hydrogen bonds between TM3 and TM7 during dynamics, which makes the two helices rotate in a cogwheel pattern as shown in Figure 12. 


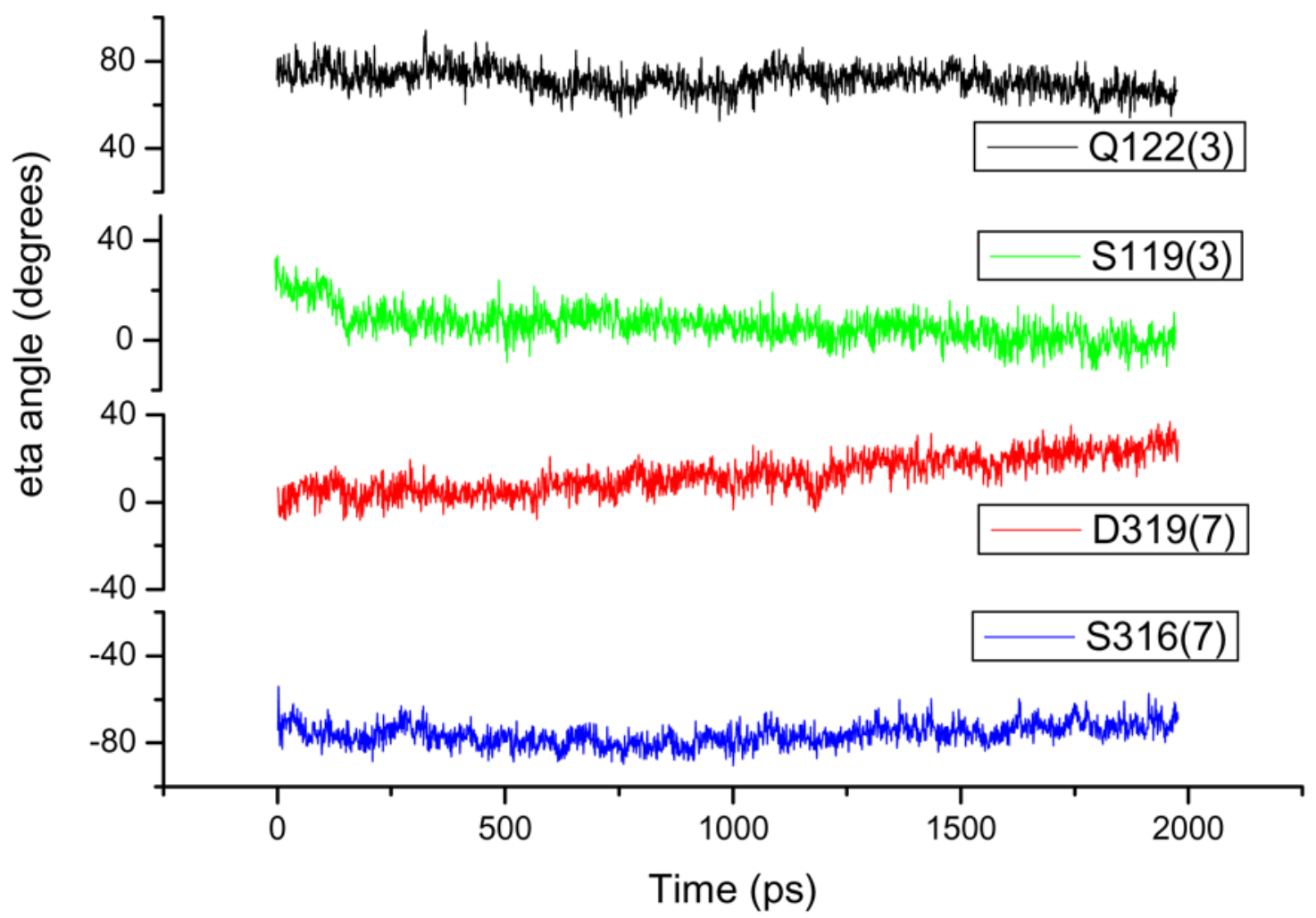



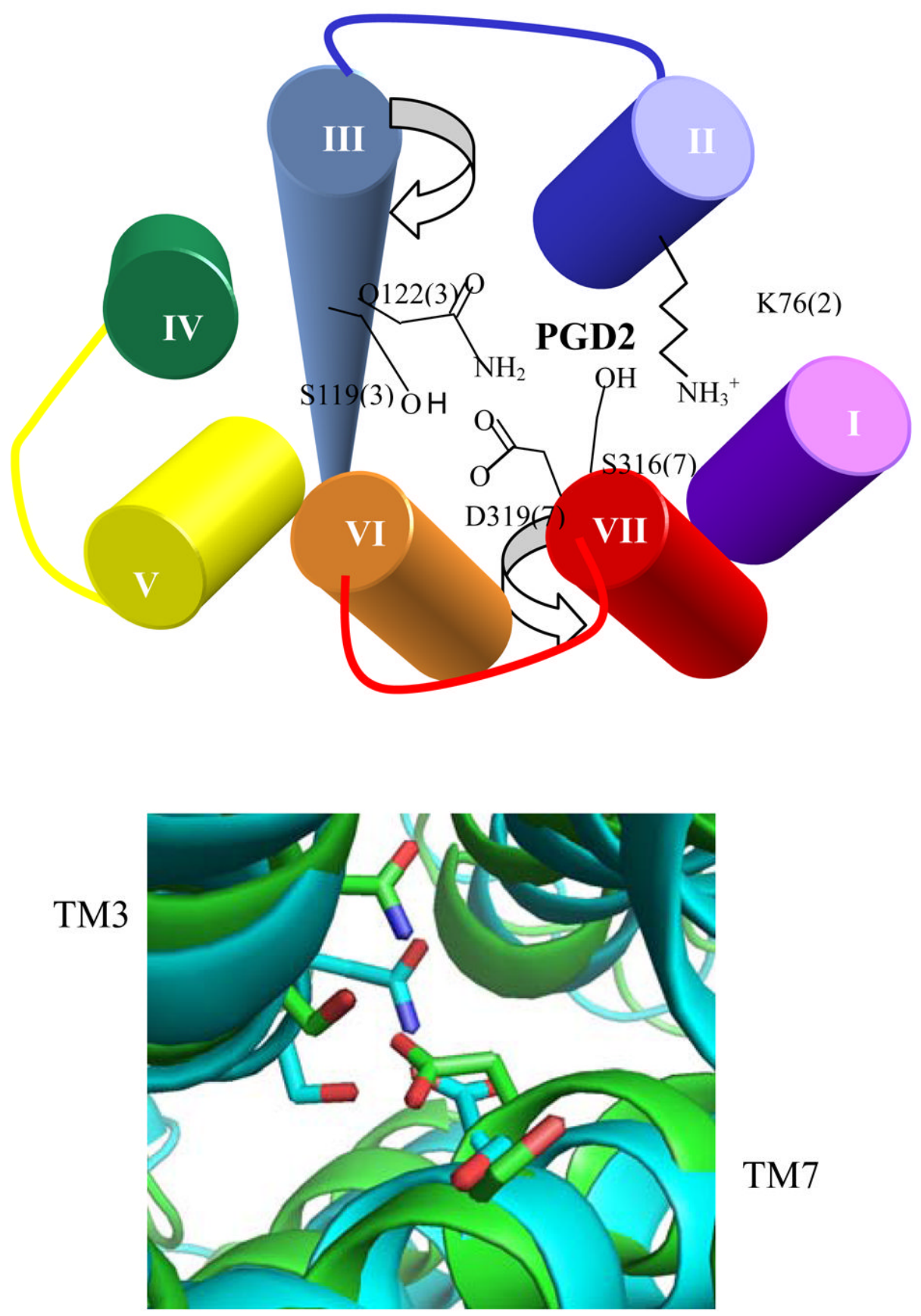

TM7

Figure 12.

Figure 12(a) The time evolution of eta angle of TM7 helix and TM3 helix. The eta angle of the residue on the helix is defined by $\mathrm{C} \alpha$ atom, Center of helix, Center of the whole protein projected to the plane perpendicular to the helix axis. We find that TM7 helix ( D319(7) and $\mathrm{S} 316(7)$ ) is rotating anticlockwise and TM3 helix is rotating clockwise in a cogwheel pattern. In other words, the eta angles of D319(7) and S316(7) are increasing. The eta angles of Q122 (3) and $S 119(3)$ are decreasing.

Figuer 12(b) The schematic representation of the rotation of TM7 helix and TM3 helix upon binding of PGD2. 
Figuer 12(c). The structure of TM3, TM7 before MD and after MD with PGD2 bound. Q122 (3), S119(3), D319(7), S316(7) are colored in green and light blue for before MD and after 2ns $\mathrm{MD}$, respectively. 


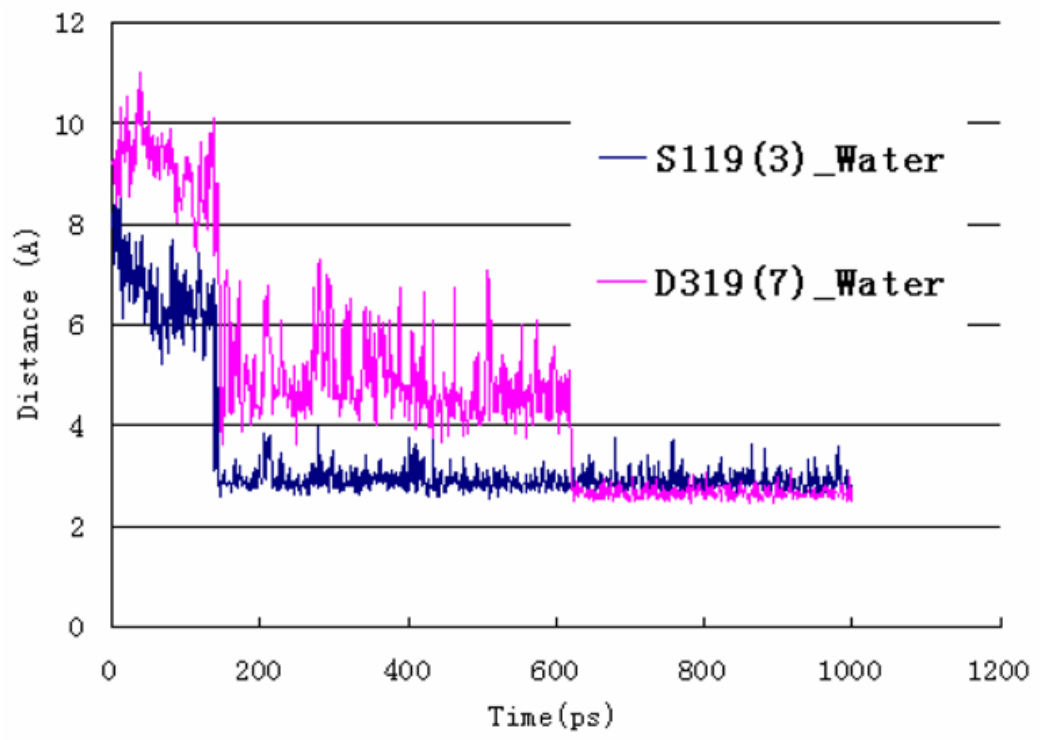

Figure 13.

After 1ns MD, we find 2 waters in the binding site for PGD2/hDP, which are close to $\mathrm{S} 119(3)$ and D319(7) respectively. 


\begin{tabular}{|c|c|}
\hline \multicolumn{2}{|l|}{ TM1 } \\
\hline $\mathrm{hDP}$ & KGNSAVMGGVLF STGLLGELLAL GLLARS \\
\hline hIP & GSVGPATSTLMFVA GVVGT̈GLAL GILSAR \\
\hline hEP1 & GASP AL P IF SMTL GA VSKLLAL ALLA QA \\
\hline $\mathrm{hEP} 2$ & P GE SPA I SSVMF SAGVLGKL I AL ALLARR \\
\hline hEP3 & GSVSVAFP ITMLLTGFVGHALAMLLVSRS \\
\hline hEP4 & LNSPVTI PAVMF IF GVVGKLVAIVVLCKS \\
\hline hF P & NRL SVFF SVI FMTVGILSHSLAIAILMKA \\
\hline $\mathrm{hTP}$ & L IASPWF AAS FCVVGL ASNLLAL SVLA GA \\
\hline \multicolumn{2}{|r|}{ ( } \\
\hline $\mathrm{hDP}$ & 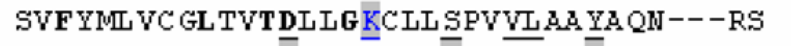 \\
\hline hIP & SAFAVLVTGLAATDLLGTSFL SPAVFFA ȲARNSSLG \\
\hline hEP1 & TTFLLFVASLLATDLAGH--VIPGALVL RU Y------ \\
\hline hEP2 & SLFHVLVTELVFTDLLGTCL I SPVVL AS YARN---QT \\
\hline hEP3 & KSFLLC IGWLALTDL VGQLLTTPVVIVVYLS---KQR \\
\hline $\mathrm{hEP} 4$ & TTFYTLVC GLAVTDLLG TLLVSPVTIATYMK----GQ \\
\hline $\mathrm{hFP}$ & ASFLLLASGLV ITDF FGHU INGAIAVFVYAS---DKE \\
\hline $\mathrm{hTP}$ & SSFL TFLC GLVLTDFLGLLVTGTIVVSQHA ALFEWHA \\
\hline \multicolumn{2}{|r|}{ 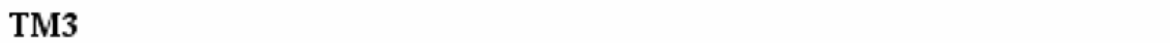 } \\
\hline $\mathrm{hDP}$ & PALD NSLC QAF AF FMSF FGLSS TL QLLAMLLE CWLSL $G$ \\
\hline hIP & PA----LC DAF AF AMTF FGLASML IL F AMA VE RCLALS \\
\hline hEP1 & PA-- GGAC HFL GGCMVF F GLCPLLLGCGMAVE RCVGVT \\
\hline hEP2 & PE--SRAC TYF AF AMTF FSLATMLML F AMLLE RYLSIG \\
\hline hEP3 & PS-- GRLC TFF GL TMTV FGLSSLF IASAMLVE RALA I R \\
\hline hEP 4 & P-----LCEYSTF ILLF FSLSGLS I I CAMSVERYLAIN \\
\hline $\mathrm{hFP}$ & QS--NVLCSIF GI CMVF SGLCPLLLGSVMA IE RC IGVT \\
\hline $\mathrm{hTP}$ & R-----LCRFMGVVM IF FGLSPLLLGAMMA SE RYLGI T \\
\hline \multicolumn{2}{|r|}{ 然 } \\
\hline $\mathrm{hDP}$ & VQYCPGTHCF I QMVHEE GSLS \\
\hline hIP & QQYCPGSWCFL RMRWAQPGG- \\
\hline hEP1 & EL QY PG TWCF I GL GP PGGWRQ \\
\hline $\mathrm{hEP} 2$ & VQYCPGTWCF I RHGR------ \\
\hline hEP3 & TVQWPGTWCF ISTGRGGNGTS \\
\hline hEP 4 & RU QYPDTWCF I DWTTWVTAH- \\
\hline hF P & KIQASRTWCFYNTED IKDWED \\
\hline $\mathrm{hTP}$ & TVQYPGSHCFL TL GAES GDVA \\
\hline \multicolumn{2}{|r|}{ 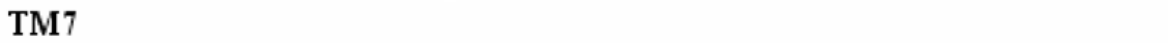 } \\
\hline $\mathrm{hDP}$ & LRAIRF LSVISIVDPH I F I IFRSPV \\
\hline hIP & LLAFRF YAF NP ILDPFV F ILFRKAV \\
\hline hEP 1 & FL AVRU ASWNQILDPFV Y I LLRQAV \\
\hline $\mathrm{hEP} 2$ & LQALRF LS I NS I IDPFV FA ILRPPV \\
\hline hEP3 & L I AVRU ASL NQILDPFV YLLLRKIL \\
\hline hEP 4 & LQA IR I ASV NP ILDPW I Y ILLRKTV \\
\hline hFP & LF ALRMATWNQILDPFV Y ILLRKAV \\
\hline $\mathrm{hTP}$ & L I YLRV ATWNQILDPFV Y ILFRRAV \\
\hline
\end{tabular}

Figure 14.

Amino acid sequence alignment of 8 human prostanoid receptors for TM1,2,3,7 and ECII. Conserved residues within the prostanoid family are in bold. The residues of DP predicted to make strong Hydrogen Bonds to PGD2 are in blue while the residues involved in hydrophobic stabilization of the PGD2 are underlined. The residues shown by mutation to be important to binding are highlighted in grey, (includes G23(1), K76(2) of DP/IP chimericas; ${ }^{29} \mathrm{D} 60(2)$, S68(2), Y75(2), F95(3), F97(3), F278(7), R279(7) of IP receptor; ${ }^{30,31}$ R302(7), L304(7), Thr (ECII) of EP2; 32 W199(ECII), T202(ECII), R329(7) of EP3; ${ }^{33-35} \mathrm{H} 81(2) ; 36,37$ and R291 $(7)^{37}$ of FP; L291(7), R295(7), W299(7) of TP38)i 


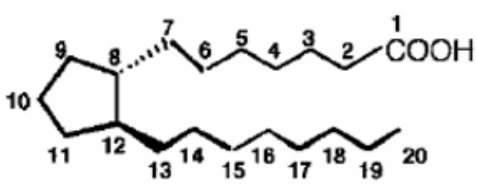

Prostanoid compound<smiles>CCCCC(O)C=CC1C(O)CC(O)C1C=CCCC(=O)O</smiles>

PGF2 $\alpha$

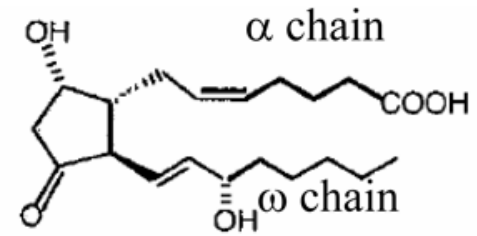

PGD2<smiles>CCCCC(O)CC=CC1C(O)CC(=O)C1CC=CCCC(=O)O</smiles>

PGE2

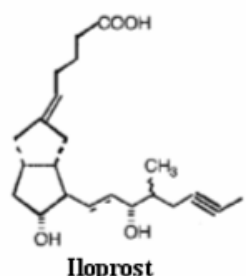

Figure 15.

Prostanoid compounds 


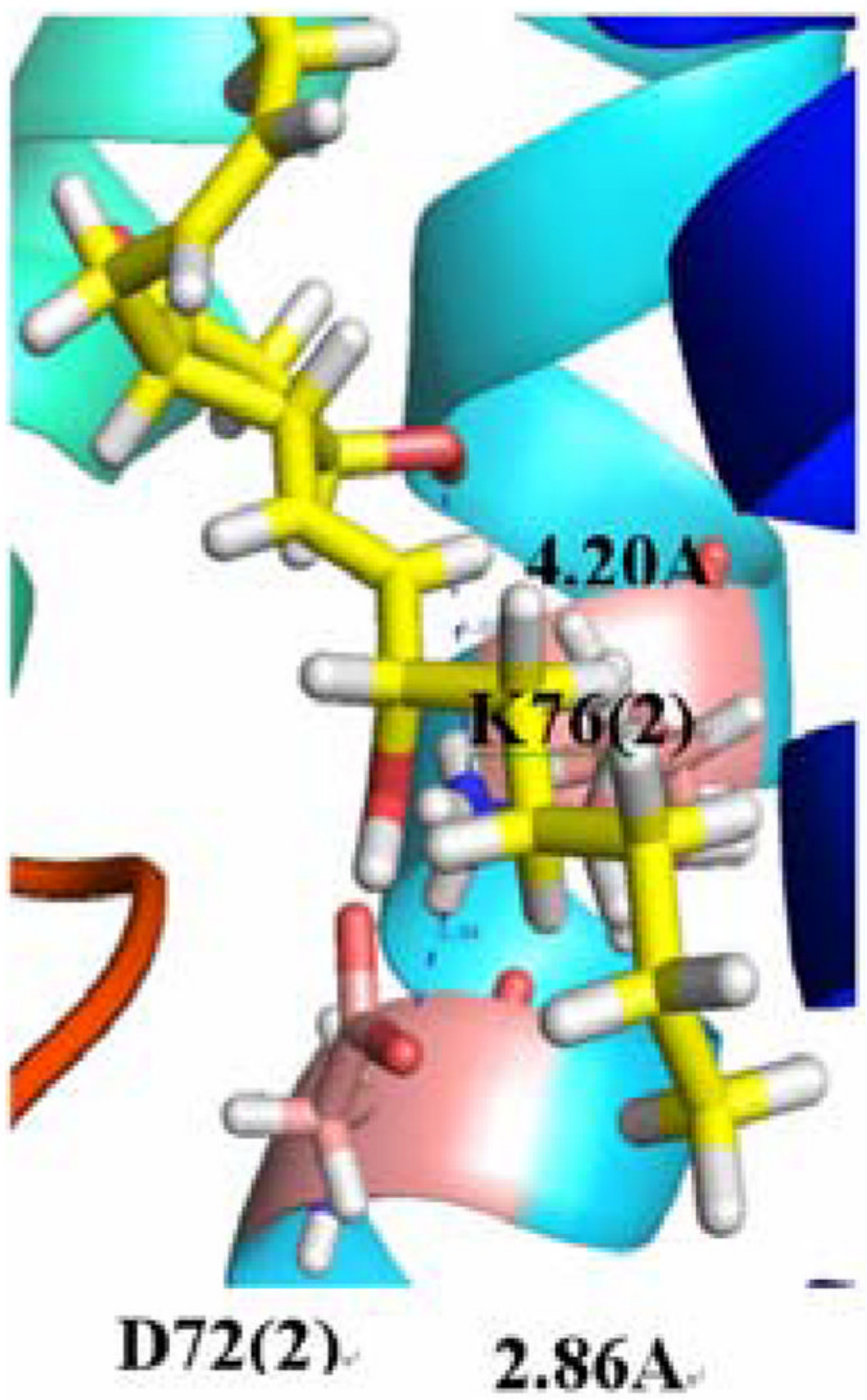

Figure 16.

PGE2 bound to hDP. In PGD2 K76(2) couples with both D72(2) and 11-CO. But in PGE2, the 11-OH points away from K76(2), while K76(2) remains bound to D72(2). 


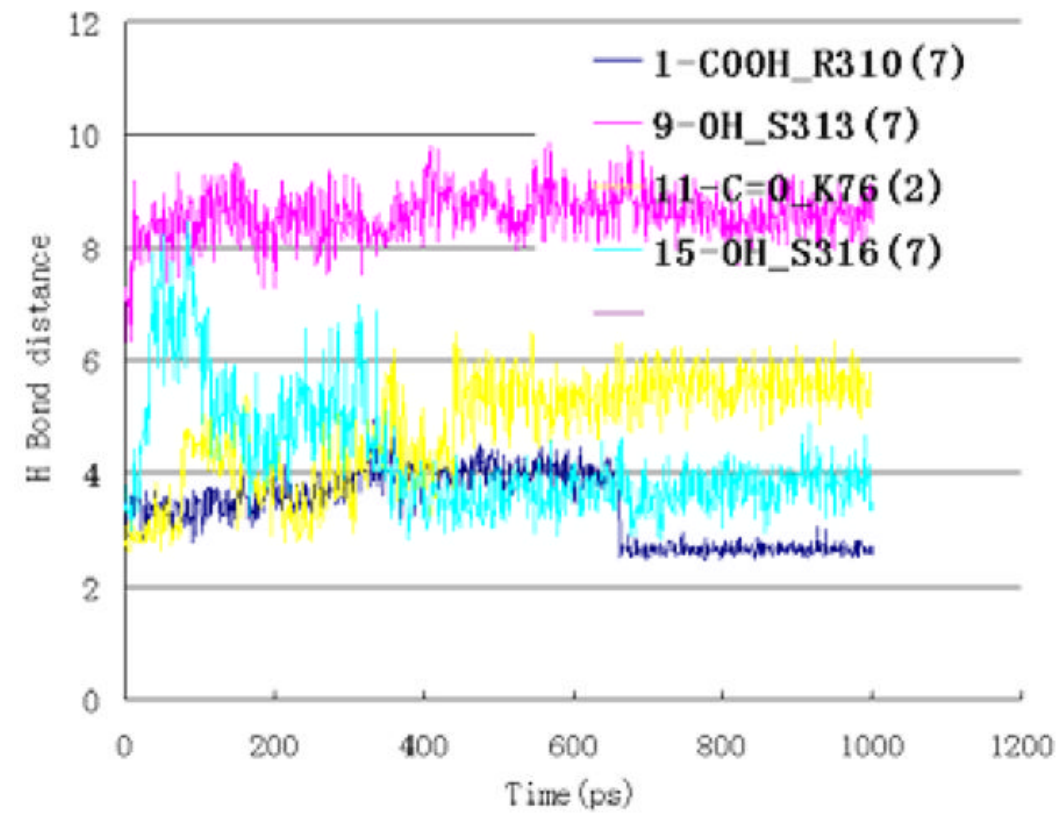

Figure 17.

Results from 1ns simulation of PGE2/hDP. The hydrogen bonds to $1-\mathrm{COOH}$ and $15-\mathrm{OH}$ are stable, while $9-\mathrm{OH}$ and $11-\mathrm{C}=\mathrm{O}$ do not form stable hydrogen bonds with $\mathrm{hDP}$ receptor. 


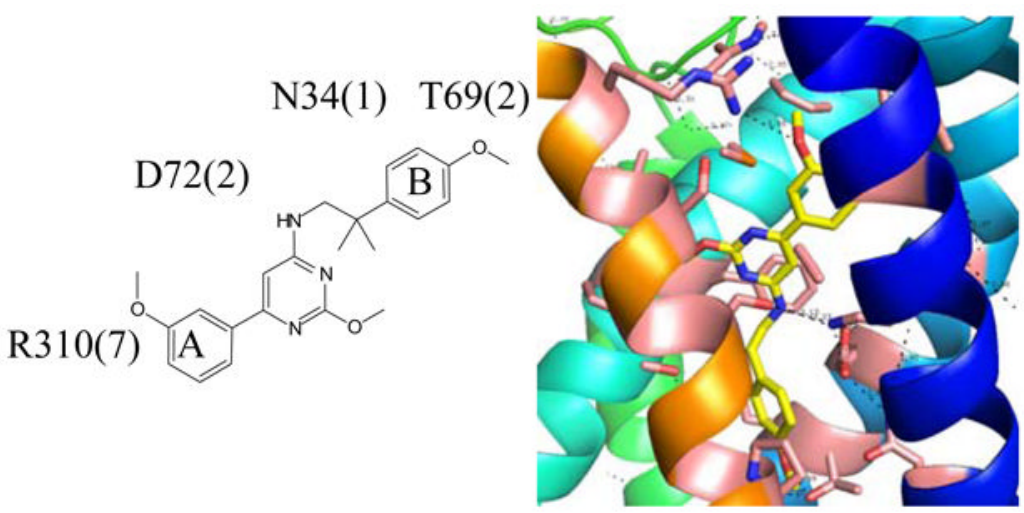

Figure 18.

The new pyrimidine antagonist and the predicted binding mode with human DP receptor. 


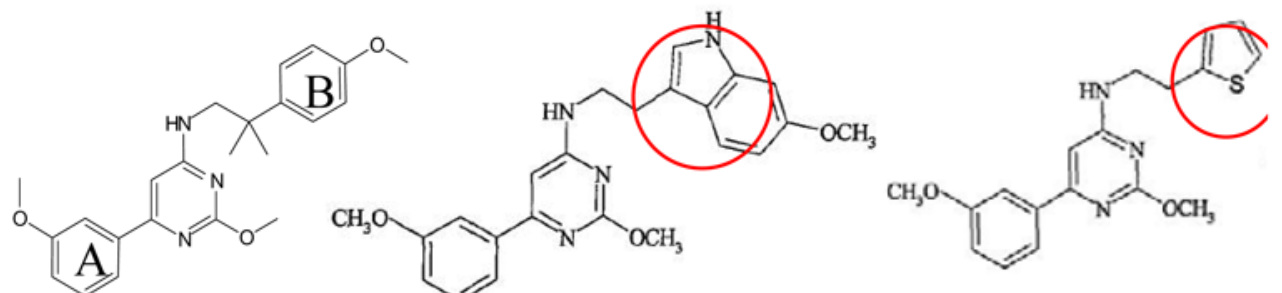
a) BE: $-56.5 \mathrm{kcal} / \mathrm{mol}$
b) BE: $-57.9 \mathrm{kcal} / \mathrm{mol}$
c) BE: $-59.5 \mathrm{kcal} / \mathrm{mo}$

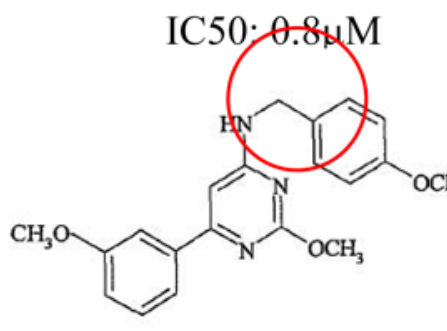

IC50: 104nM

IC50: $9.7 \mathrm{nM}$

d) BE: $-54.8 \mathrm{kcal} / \mathrm{mol}$

e) BE: $-62.7 \mathrm{kcal} / \mathrm{mol}$

f) BE: $-58.9 \mathrm{kcal} / \mathrm{mol}$

IC50: 1073nM

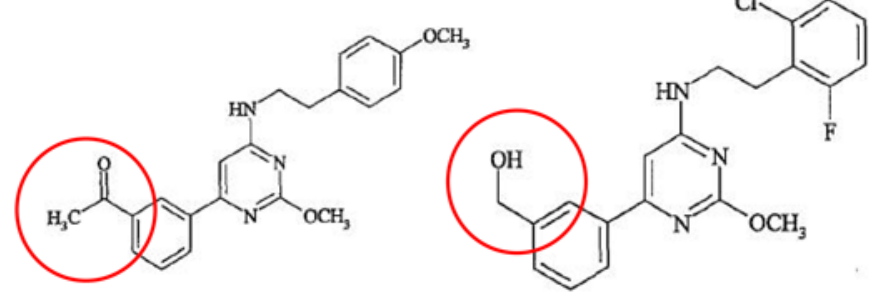

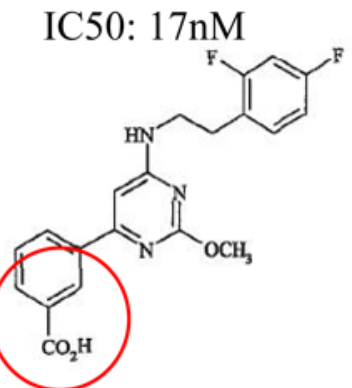

g) BE: $-61.1 \mathrm{kcal} / \mathrm{mol}$

h) BE: $-62.8 \mathrm{kcal} / \mathrm{mol}$

i) $\mathrm{BE}:-80.6 \mathrm{kcal} / \mathrm{mol}$

IC50: $5 \mathrm{nM}$

IC50: $2.4 \mathrm{nM}$

IC50: 0.8nM

Figure 19.

The modified pyrimidine compounds, all based on molecule 19a, shown in Fig. 18 